\title{
Chain Conformation Control of Fluorene-Benzothiadiazole Copolymer Light-Emitting Diode Efficiency and Lifetime
}

\author{
Bingjun Wang, Hao Ye, Moritz Riede, and Donal D. C. Bradley* \\ Cite This: https://dx.doi.org/10.1021/acsami.0c18490 \\ Read Online
}

ABSTRACT: The $\beta$-phase, in which the intermonomer torsion angle of a fraction of chain segments approaches $\sim 180^{\circ}$, is an intriguing conformational microstructure of the widely studied light-emitting polymer poly(9,9-dioctylfluorene) (PFO). Its generation can in turn be used to significantly improve the performance of PFO emission-layer-based light-emitting diodes (LEDs). Here, we report the generation of $\beta$-phase chain segments in a copolymer, 90F8:10BT, containing 90\% 9,9-dioctylfluorene (F8) and 10\% 2,1,3-benzothiadiazole (BT) units and show that

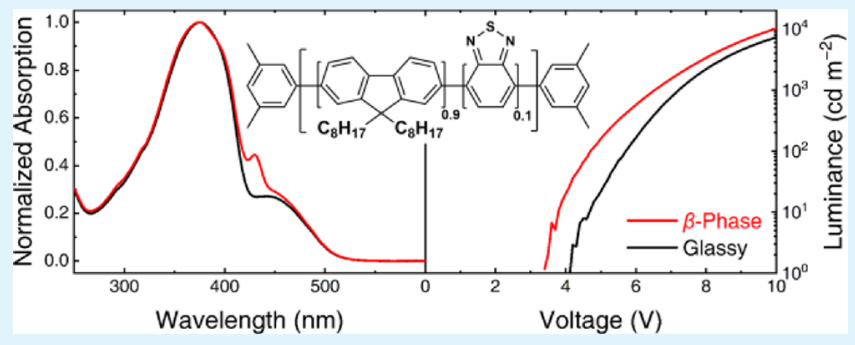
significant improvements in performance also ensue for LEDs with

$\beta$-phase 90F8:10BT emission layers, generalizing the earlier PFO results. The $\beta$-phase was induced by both solvent vapor annealing and dipping copolymer thin films into a solvent/nonsolvent mixture. Subsequent absorption spectra show the characteristic fluorene $\beta$-phase peak at $\sim 435 \mathrm{~nm}$, but luminescence spectra $(\sim 530 \mathrm{~nm}$ peak) and quantum yields barely change, with the emission arising following efficient energy transfer to the lowest-lying excited states localized in the vicinity of the BT units. For $\sim 5 \% \beta$-phase chain segment fraction relative to $0 \% \beta$-phase, the LED luminance at $10 \mathrm{~V}$ increased by $\sim 25 \%$ to $5940 \mathrm{~cd} \mathrm{~m}^{-2}$, the maximum external quantum efficiency by $\sim 61$ to $1.91 \%$, and the operational stability from $64 \%$ luminance retention after $20 \mathrm{~h}$ of operation to $90 \%$. Detailed studies addressing the underlying device physics identify a reduced hole injection barrier, higher hole mobility, correspondingly more balanced electron and hole charge transport, and decreased carrier trapping as the dominant factors. These results confirm the effectiveness of chain conformation control for fluorene-based homo- and copolymer device optimization.

KEYWORDS: $\beta$-phase, chain conformation, polymer light-emitting diodes, polyfluorenes, copolymers

\section{INTRODUCTION}

Following the first report of a solution-processed conjugated polymer light-emitting diode (LED) in $1990,{ }^{1}$ the past three decades have seen the successful commercial development of ink-jet-printed high-resolution LED displays, with these devices also expected to play an increasing role in solid-state lighting applications where high-throughput manufacturing at suitably low cost is required. In addition to their solution processability, polymer semiconductors lend themselves to physical structure control via manipulation of chain conformation, ${ }^{2}$ liquid crystalline, ${ }^{3,4}$ and crystalline ordering. ${ }^{5}$ This provides novel possibilities to optimize structure-propertydevice performance relationships and with the right processing methods yields innovative patterning approaches for electronic, optoelectronic, and photonic devices. ${ }^{6-10}$ Among such polymers, poly(9,9-dioctylfluorene) (PFO) is one of the most widely studied, and it has been proven to be an excellent model system that allows the exploration of physical structure control. This, combined with a relatively short wavelength luminescence emission, has made PFO the baseline blue polymer LED emission material. ${ }^{7,11,12}$

The $\beta$-phase microstructure of $\mathrm{PFO}^{8,9,13}$ comprises a fraction of chain segments for which the intermonomer torsion angle, $\varphi$, approaches $180^{\circ}$, resulting in the adoption of a chain extended planar conformation with alkyl substituent groups for neighboring fluorene units on opposite sides of the chain (Figure 1a). It differs from the normal glassy phase obtained by spin-coating from a good solvent, where the average angle $\langle\varphi\rangle \approx 135^{\circ} .{ }^{14}$ Generation of the $\beta$-phase
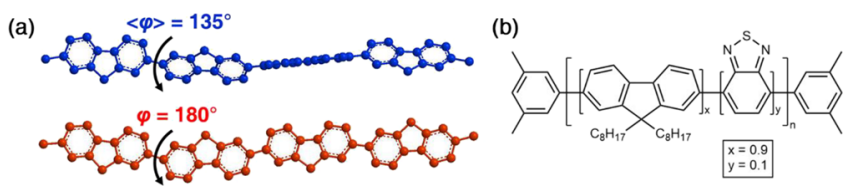

Figure 1. (a) Schematic PFO chain conformations in the glassy (blue) and $\beta$ - (red) phases. Note that the hydrogen atoms and dioctyl side chains have been omitted for clarity. (b) The chemical structure of 90F8:10BT.

Received: October 14, 2020

Accepted: December 14, 2020 
requires different fabrication conditions either during film deposition or via additional treatment of glassy samples (spincoated or quenched from the nematic melt). Examples of the former include using moderate/poor ${ }^{4,13}$ and/or high boiling point $^{15}$ solvents, inclusion of high-boiling-point additives, ${ }^{16,17}$ and Langmuir-Blodgett deposition. ${ }^{18}$ The latter category includes thermal cycling to liquid nitrogen temperature and back to room temperature, ${ }^{4,13,19}$ solvent vapor annealing (SVA), ${ }^{20,21}$ writing solvent on top of the film, ${ }^{22}$ and dipping the glassy sample in a solvent/nonsolvent mixture (or simply "dipping" for short). ${ }^{21,23}$ The presence of the $\beta$-phase can be readily confirmed from characteristic changes in optical properties, ${ }^{13-15,19,21}$ comprising the appearance of a new narrow-linewidth absorption peak at $\sim 435 \mathrm{~nm}$, and a red-shift and relative intensity enhancement of the main $\left(S_{1} \rightarrow S_{0} 0-0\right)$ photoluminescence (PL) peak. This shift from $\sim 420$ to $\sim 440$ $\mathrm{nm}$ is also accompanied by an increase in vibronic peak resolution due to a narrowing of the peaks. We emphasize that for " $\beta$-phase samples" or " $\beta$-phase devices", a fraction of $\varphi \approx$ $180^{\circ}$ chain-extended segments are embedded within an otherwise glassy matrix, where the fraction can be varied from 0 (i.e., fully glassy) to about $45 \%,{ }^{16}$ although at this latter percentage, scattering films with poor optical quality typically occur. In our own experience, $\sim 25 \%$ is the limit to maintain good film quality. ${ }^{21}$

Initial interest in the $\beta$-phase in PFO was from the perspective of better understanding the influence of the physical structure on photophysical properties ${ }^{5,19,21,24}$ and as a "self-doped" donor-acceptor system, ${ }^{4}$ which provides an excellent platform to investigate fundamental topics in energy transfer and excitation dynamics. ${ }^{15,25,26}$ Subsequent interest broadened to include refractive index patterning $8,20,22$ for photonic structure fabrication and also device optimization. In the latter category, a small $\beta$-phase fraction of $\sim 1.3 \%$ was shown to lead to a large increase in the luminance and external quantum efficiency (EQE) of PFO LEDs. ${ }^{23}$ In addition, $\beta$ phase $\mathrm{PFO}$ was found to be a promising gain medium and to control the polariton physics of metal-PFO-metal microcavities.'

Motivated by the attractive features of $\beta$-phase conformational control of electronic, optoelectronic, and photonic properties in PFO, researchers have sought to broaden the range of materials within which a $\beta$-phase-like change in the physical structure might be induced. PFO homologs with heptyl and nonyl substituents were shown to support $\beta$-phase formation, while hexyl and decyl substituents suppressed $\beta$ phase formation. ${ }^{27}$ Copolymers of 9,9-dioctylfluorene (F8) with low fractions $(\leq 10-15 \%)$ of dibenzothiophene-S,Sdioxide and dibenzothiophene also show $\beta$-phase formation but have been little studied. ${ }^{28}$ Likewise, the $\beta$-phase was observed in electron-beam cross-linkable alternating copolymers of dihexylfluorene and vinyl-ether-functionalized dialkyl fluorene. ${ }^{29}$ More recently, the $\beta$-phase has been generated successfully in F8-based copolymers 97F8:3BSP, 95F8:5BSP, and 90F8:10BSP, containing, respectively, 3, 5, and 10\% butylsubstituted phenylenediamine (BSP) units. $^{30}$ In contrast, 80F8:20BSP did not allow $\beta$-phase generation, with the F8 sequence length being too short. The key benefit of forming the $\beta$-phase in 95F8:5BSP emission layer (EML) LEDs was to improve the device CIE $(x, y)$ color coordinates from $(0.149$, $0.175)$ to $(0.145,0.123)$, achieving a significantly deeper-blue emission with outstanding spectral stability. This results from the localization of excited states on the $\beta$-phase F8 segments instead of arylamine moieties, thereby changing the emission from a broad, red-shifted CT-exciton-like band to predominantly being a vibronically structured neutral exciton emission, characteristic of $\beta$-phase PFO.

In the current study, we investigate $\beta$-phase generation and LED performance for a 90F8:10BT copolymer comprising 90\% F8 and 10\% 2,1,3-benzothiadiazole (BT) units (Figure 1b). In this copolymer, the lowest-lying BT absorption band overlaps the emission spectrum of the F8 segments, leading to efficient Förster energy transfer prior to luminescence emission, just as also observed for LED-optimized "5BT:95F8" polymer blends, which comprise 5\% poly $(9,9$ dioctylfuorene-alt-2,1,3-benzothiadiazole) (F8BT) and 95\% PFO, yielding an overall $2.5 \%$ fraction of BT units. ${ }^{31}$ The resulting emission then lies in the green spectral range, characteristic of BT-containing polymers and starburst molecules. ${ }^{32}$ The 90F8:10BT copolymer has previously been used in optically pumped lasers, ${ }^{33}$ optical amplifiers, ${ }^{34}$ and quantum-dot-enhanced LEDs, ${ }^{35}$ but in none of those studies was consideration given to the effects of $\beta$-phase formation. Herein, we report the generation of $\beta$-phase F8 chain segments in 90F8:10BT and demonstrate resulting improvements in performance for 90F8:10BT EML LEDs. For devices with a $\sim 5 \% \beta$-phase fraction induced by dipping, the luminance (at $10 \mathrm{~V}$ ) and the maximum EQE were improved by 25 and $61 \%$, respectively. In addition, the device stability significantly improved, yielding 90\% luminance retention after $20 \mathrm{~h}$ of continuous operation from a starting luminance of $\sim 3700 \mathrm{~cd}$ $\mathrm{m}^{-2}$. The basis for these enhancements was studied in detail, and it was found that lowering of the hole injection barrier, an increase in hole mobility leading to correspondingly more balanced charge transport, and a reduced trap density during device operation all contribute.

\section{RESULTS AND DISCUSSION}

$\beta$-Phase Generation and Resulting Optical Spectra. The $\beta$-phase was induced in 90F8:10BT thin films by both SVA and dipping. Toluene vapor was used in the former case, and a cyclohexane (solvent)/isopropanol (nonsolvent) mixture was chosen for the latter case; further details are provided in the Experimental Section. Formation of the $\beta$-phase is confirmed by the appearance of the characteristic $\beta$-phase peak $^{13,15,21}$ at $\sim 430 \mathrm{~nm}$ in the absorption spectra (Figure 2a) following both SVA and dipping. The $\beta$-phase chain segment fraction (listed in Table 1) can then be estimated from the difference between glassy and $\beta$-phase spectra, taking into account the difference in the oscillator strengths for glassy and $\beta$-phase chain segments (Figure S1). ${ }^{21}$ The SVA method yields a higher fraction of $\beta$-phase segments, although in both cases the glassy phase remains predominant.

For glassy 90F8:10BT thin films, the main absorption peaks at $\sim 375 \mathrm{~nm}$, close to the lowest energy $S_{1} \leftarrow S_{0}$ absorption band in PFO $(390 \mathrm{~nm}) .{ }^{13,21}$ The second longer wavelength peak at $\sim 445 \mathrm{~nm}$ is attributed to the presence of the BT units, consistent with the $455 \mathrm{~nm}$ peak in the F8BT absorption spectrum. $^{36,37}$ This BT-moiety-centered contribution is also seen in truxene-cored starburst molecules with $20 \% \mathrm{BT} / 80 \%$ 9,9-dihexylfluorene side arms, appearing as a long-wavelength peak/shoulder close to $450 \mathrm{~nm}$. ${ }^{32}$ It is associated with the dipole-allowed optical transition between a delocalized highest occupied molecular orbital (HOMO) and a BT-localized lowest unoccupied molecular orbital (LUMO). ${ }^{38}$ At low BT moiety content, the LUMO is relatively destabilized, through 
(a)

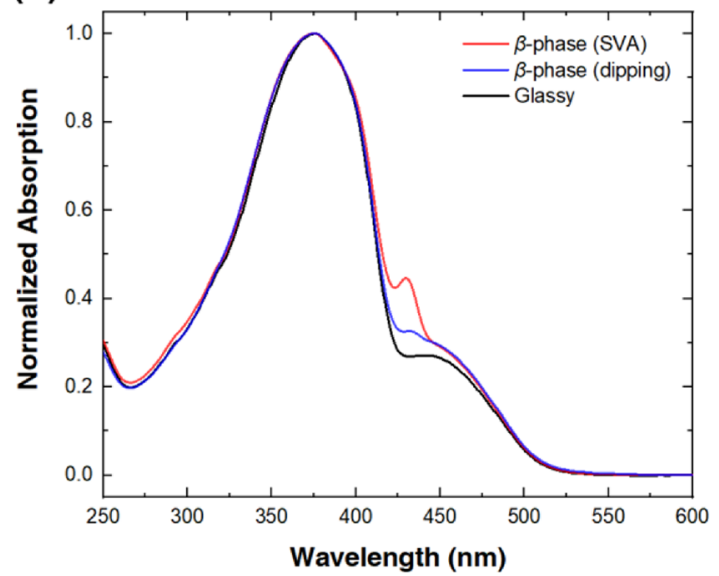

(b)

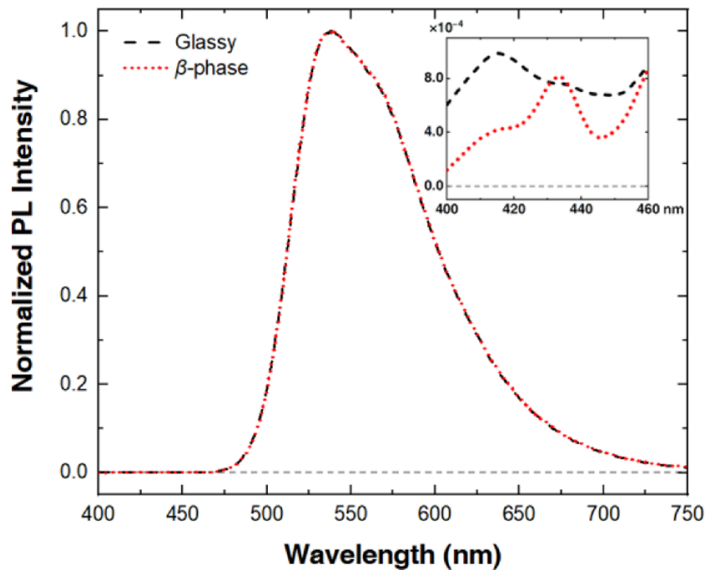

Figure 2. (a) Normalized absorption and (b) PL spectra for the glassy and $\beta$-phase 90F8:10BT samples. The inset to panel (b) shows the PL spectra from 400 to $460 \mathrm{~nm}$ on an expanded scale. Glassy-phase absorption is plotted as a black line, and $\beta$-phase, generated by SVA and dipping, is plotted as red and blue lines, respectively. For PL, the glassy spectrum is plotted as a dashed black line, and the $\beta$-phase (SVA) spectrum is plotted as a dotted red line; the gray dashed line is the zero PL intensity level.

Table 1. $\beta$-Phase Fraction and PLQE Values of the Glassy and $\beta$-Phase 90F8:10BT Samples

\begin{tabular}{lcc}
\multicolumn{1}{c}{ sample } & $\beta$-phase fraction (\%) & PLQE (\%) \\
glassy & 0 & $80 \pm 5$ \\
$\beta$-phase (SVA) & $8 \pm 1$ & $80 \pm 5$ \\
$\beta$-phase (dipping) & $5 \pm 1$ & $80 \pm 5$ \\
\hline
\end{tabular}

there being little BT-to-BT interaction, and as a consequence, the band is blue-shifted relative to F8BT. ${ }^{38}$

The $375 \mathrm{~nm}$ absorption band derives instead from the same delocalized HOMO, but the transition is to higher-lying unoccupied molecular orbitals that have electron density largely on the fluorene moieties. The observed blue-shift of this peak relative to PFO has been postulated, in light of quantum chemical calculations, to arise from a destabilization of the associated unoccupied molecular orbitals through the effect that the BT moieties have on limiting the F8 sequence/ conjugation length, just as occurs for oligomers with lower repeat unit numbers. ${ }^{38}$

Figure $2 \mathrm{~b}$ shows the PL spectra of glassy and $\beta$-phase 90F8:10BT thin-film samples excited at $380 \mathrm{~nm}$, close to the extended F8 sequence centered absorption peak. The PL emission spectra are largely independent of the presence of $\beta$ phase chain segments and their generation method; the data shown here are for a film subjected to SVA. There is a single dominant emission band peaked at $538 \mathrm{~nm}$, which is very similar to that of F8BT ( $540 \mathrm{~nm}$ peak). ${ }^{36,37}$ Expanding the intensity scale in the 400 to $460 \mathrm{~nm}$ spectral region (Figure $2 \mathrm{~b}$ inset) reveals weak features that show a dependence on the presence or otherwise of $\beta$-phase chain segments. For the glassy sample, there is a peak located at $\sim 415 \mathrm{~nm}$, whereas for the $\beta$-phase sample, the strongest peak is at $\sim 435 \mathrm{~nm}$ with a shoulder at $\sim 415 \mathrm{~nm}$. These are close to the characteristic emission peaks for glassy and $\beta$-phase PFO at $\sim 420$ and $\sim 440$ $\mathrm{nm}^{13,21,24}$ and are, therefore, assigned to residual emission from a small fraction of excitons that avoid transfer to BTcentered excited states. The F8-to-BT excited-state energy transfer process is evidently very efficient, leading to a 3 orders of magnitude higher steady-state emission intensity from BTcentered states. This is despite the BT moieties comprising only $10 \%$ of the polymer backbone units. As a result, whether the extended F8 backbone sequences in a 90F8:10BT film are in the glassy or $\beta$-phase does not significantly alter the steadystate $\mathrm{PL}$ emission spectrum. This contrasts with previous results $^{38}$ for a range of $x \mathrm{~F} 8: y \mathrm{BT}$ polymers where $\mathrm{F} 8$ emission is still clearly visible in the PL spectrum for BT fractions up to $26 \%(x=74, y=26)$, but it should be noted that those measurements were performed for dilute solution samples. It is evident that energy transfer is much more effective in the solid state, likely a combined effect of more extended, rigid, and densely packed polymer chains.

One of the important parameters for EML materials is the photoluminescence quantum efficiency (PLQE), which enumerates the relative efficiency of radiative decay. Table 1 summarizes the 90F8:10BT thin-film PLQE values for the glassy and $\beta$-phase samples (generated by both SVA and dipping), which all show a high PLQE of $80 \pm 5 \%$. This is reasonably consistent with reported values ${ }^{38}$ although in that case the measurements were performed for dilute solutions and the consequent less-efficient energy transfer yields PLQE values that are closer to PFO than F8BT. For PFO thin films, the PLQE ranges from 50 to $80 \%$ depending on the state of order (spin-coated glassy, $\beta$-phase, crystalline, or nematic glassy). ${ }^{5,21}$ For F8BT, the PLQE has been reported to lie between $58 \pm 5 \%$ for thin films ${ }^{39}$ and $78 \%$ for dilute solutions. $^{38}$

In addition to measuring the PLQE values, we have also recorded PL decay transients to assess the radiative exciton lifetimes for thin-film samples with and without the $\beta$-phase. Figure 3 shows the transient PL results as well as the extracted lifetimes, derived from single exponential fits, with $R^{2}>0.995$. The lifetimes are close to but slightly longer than that for F8BT (2.03 ns) and substantially longer than for PFO (227 ps). ${ }^{39}$ The single exponential decay and the long lifetime are consistent with (i) the exciton being localized in the vicinity of the BT units (as also deduced from the spectral data) and (ii) energy transfer from extended F8 sequences to BT sites being highly efficient and much more rapid than the subsequent decay. The excitons in 90F8:10BT additionally have an $\sim 9 \%$ shorter lifetime when the $\beta$-phase is present. Given that the PLQE values show no significant difference between the glassy and $\beta$-phase samples (Table 1 ), the decrease in lifetime is likely due to an increase in oscillator strength, consistent with the expected increase in conjugation length. ${ }^{40}$ 


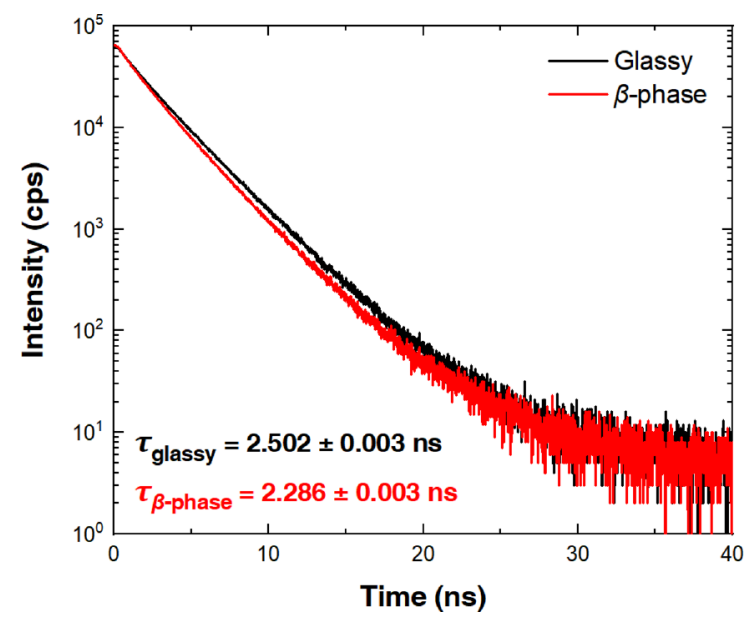

Figure 3. Transient PL decay curves of glassy (black) and $\beta$-phase (red) $90 \mathrm{~F} 8: 10 \mathrm{BT}$ thin films. The decay time is extracted from a single exponential fit $\left(R^{2}>0.995\right)$.

Given again that there is no change in PLQE when the $\beta$ phase is generated, it might be expected that no improvement in LED performance would be engendered by this conformation change. However, as shown in the next section, generation of $\beta$-phase chain segments is significantly advantageous to the performance of 90F8:10BT-based LEDs, through the effect that they have on charge carrier injection and transport properties.

LED Performance. To examine the influence of the $\beta$ phase in 90F8:10BT on LED performance, we fabricated devices with a conventional bottom emission structure (Figure 4a and Experimental Section). The ITO-on-glass substrate was coated with PEDOT:PSS to form a composite anode. This in turn was coated with an electron blocking interlayer comprising $\sim 15 \mathrm{~nm}$ of poly(9,9-dioctylfluorene-alt- $N$-(4-secbutylphenyl)diphenylamine) (TFB), which also reduces exciton quenching by the electrode. ${ }^{41}$ The 90F8:10BT EML coated on top was of $\sim 80 \mathrm{~nm}$ thickness, and the $\beta$-phase was generated in this film via the dipping method in order to avoid the high surface roughness that SVA can produce (Figure S2). Such roughness is undesirable as it can lead to nonuniform emission and a greater tendency for dielectric breakdown. The top metal cathode was fabricated by sequential evaporation of $\mathrm{LiF}(1 \mathrm{~nm})$ and $\mathrm{Al}(100 \mathrm{~nm})$. The expected energy level diagram is shown in Figure $4 \mathrm{~b}$ using literature values for the electron affinities, ionization potentials, and work functions. $^{30,35,42}$
Figure 5 presents device characteristics for both glassy and $\beta$-phase 90F8:10BT LEDs, which have the same emission spectrum (Figure 9) peaked at $\sim 535 \mathrm{~nm}$ in green. Figure 5a,b shows the current density $(J)$ and luminance $(L)$ versus voltage $(V)$ data, respectively. The current densities at $10 \mathrm{~V}$ are similar, with a slightly higher value for the $\beta$-phase LEDs, but the current turns on strongly at a significantly lower voltage in the latter case. The $L-V$ curves show that the strong current turnon correlates with light emission and the $\beta$-phase 90F8:10BT LEDs have higher luminance all the way up to $10 \mathrm{~V}$. The turnon voltage (the voltage at which $L=1 \mathrm{~cd} \mathrm{~m}^{-2}$ ) drops from 4.1 $\mathrm{V}$ for glassy to $3.4 \mathrm{~V}$ for the $\beta$-phase, and the luminance at $10 \mathrm{~V}$ increases from $\sim 7000$ to $>10,000 \mathrm{~cd} \mathrm{~m}^{-2}$.

Figure $5 \mathrm{c}, \mathrm{d}$ shows the EQE, luminous efficiency $\left(\eta_{\mathrm{L}}\right)$, and luminous power efficiency $\left(\eta_{\mathrm{LP}}\right)$ as a function of luminance for both devices. The peak EQE values for $\beta$-phase LEDs reach $\sim 2.4 \%$ for low luminance and remain above $1 \%$ for luminance up to $10,000 \mathrm{~cd} \mathrm{~m}^{-2}$. For glassy devices, the maximum EQE, also at low luminance, is $\sim 1.5 \%$. The luminous and luminous power efficiencies, $\eta_{\mathrm{L}}$ and $\eta_{\mathrm{LP}}{ }^{43}$ show similar trends; for $\beta$ -

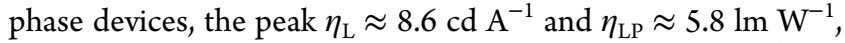
while for the glassy device, peak values are $\eta_{\mathrm{L}} \approx 5.5 \mathrm{~cd} \mathrm{~A}^{-1}$ and $\eta_{\mathrm{LP}} \approx 2.9 \mathrm{~lm} \mathrm{~W}^{-1}$.

Generation of the $\beta$-phase in another PFO-based copolymer $^{30}$ and PFO itself ${ }^{44}$ has been reported to improve operational stability, so we have also undertaken an accelerated lifetime test for the 90F8:10BT LEDs. The measurement was carried out using encapsulated devices held at constant current density $J=80 \mathrm{~mA} \mathrm{~cm}{ }^{-2}$ under ambient conditions. The initial luminance values for the glassy and $\beta$-phase devices were $\sim 2800$ and $\sim 3700 \mathrm{~cd} \mathrm{~m}^{-2}$, respectively.

Figure $5 \mathrm{e}$ shows fractional luminance retention as a function of time. After $20 \mathrm{~h}$ of continuous operation, the luminance of $\beta$-phase devices still exceeds $90 \%$ of their initial value. However, for glassy devices, $\sim 35 \%$ of the starting luminance was lost over the same period. Both devices see a fast, initial decay followed by a more gradual decay with an approximately constant slope. The $\beta$-phase devices decay less during the initial rapid drop and show a shallower slope thereafter. In addition, after about $14 \mathrm{~h}$, the glassy devices abruptly degrade with a noisy luminance fluctuation superimposed on top of the sharp decay. The time for the device luminance to drop to $90 \%$ of its initial value is denoted $\mathrm{LT}_{90}$, and we find $\mathrm{LT}_{90}=10.4 \mathrm{~h}$ for the glassy devices and $\mathrm{LT}_{90}>20 \mathrm{~h}$ for the $\beta$-phase devices. Therefore, we conclude that, as previously reported, device operational stability is significantly improved by introduction of $\beta$-phase chain segments. (a)

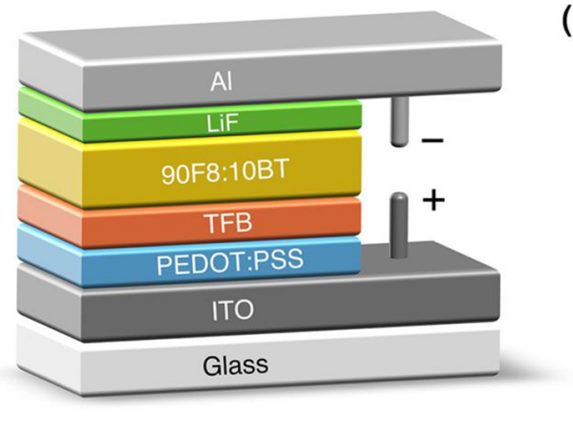

(b)

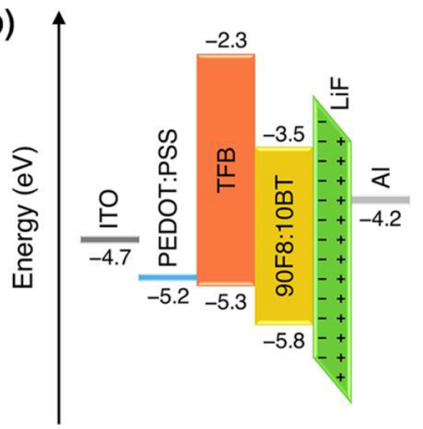

Figure 4. (a) Schematic 90F8:10BT bottom emission LED structure and (b) corresponding energy level diagram. 

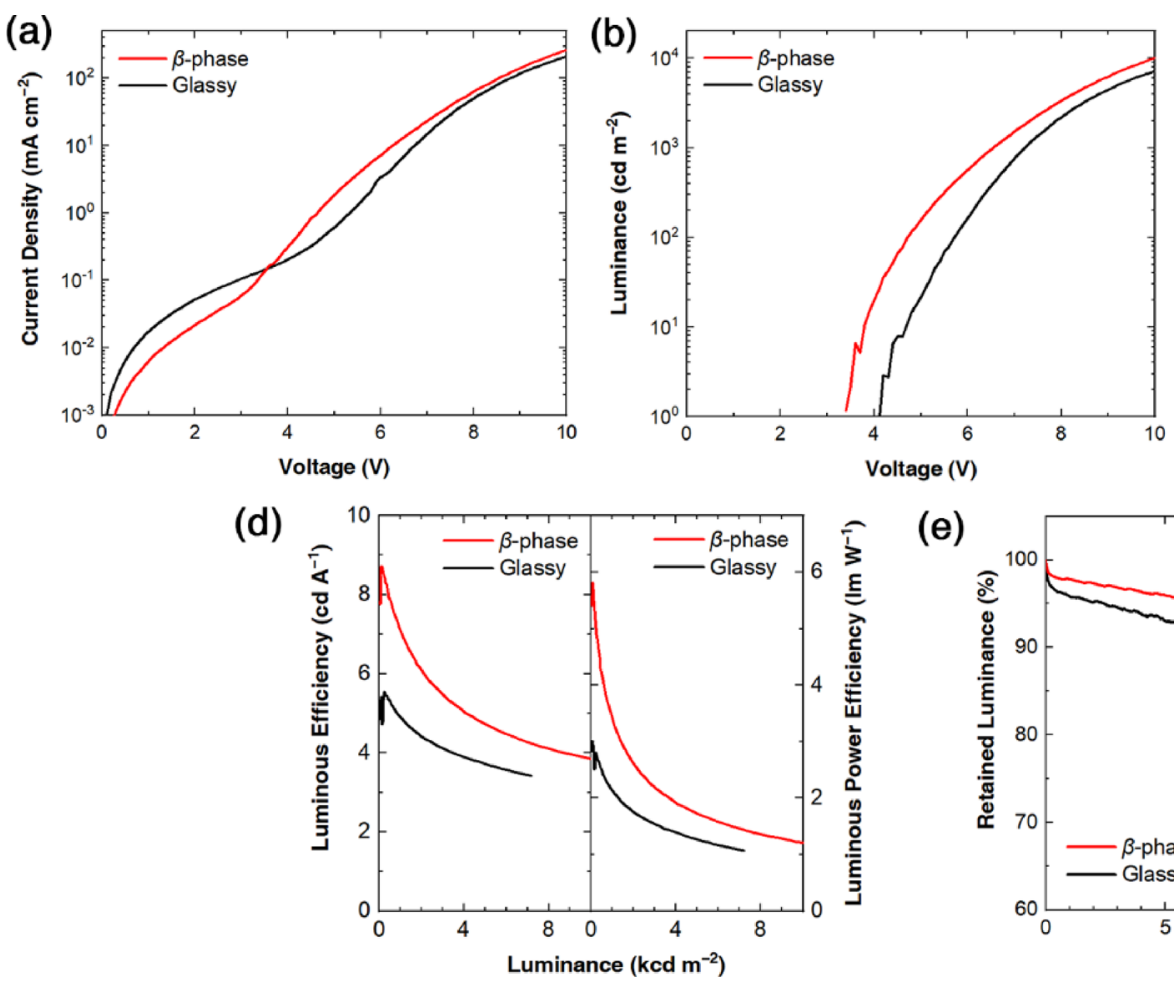

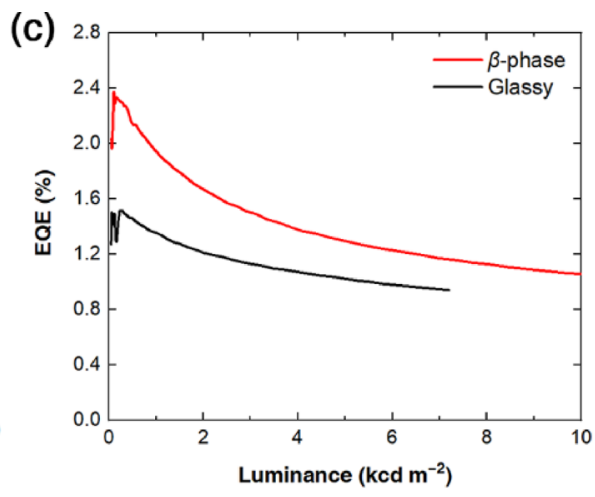

(e)

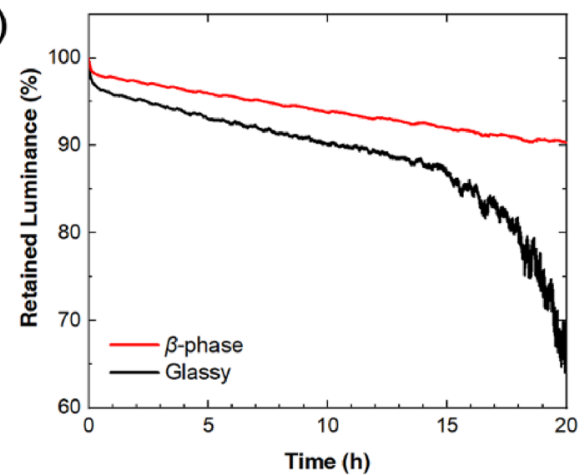

Figure 5. Typical device performance of glassy (black) and $\beta$-phase (red) 90F8:10BT LEDs. (a) $J-V$ characteristics; (b) $L-V$ characteristics; (c) EQE and (d) luminous efficiency and luminous power efficiency, as a function of luminance; (e) accelerated device lifetime test results, performed on encapsulated devices held at constant current density $J=80 \mathrm{~mA} \mathrm{~cm}^{-2}$.

Table 2 summarizes the average performance parameters for more than 20 devices of each type. It is evident that generating

Table 2. Average Device Performance and Lifetime of Glassy and $\beta$-Phase 90F8:10BT LEDs

\begin{tabular}{|c|c|c|c|}
\hline & glassy device & $\begin{array}{c}\beta \text {-phase } \\
\text { device }\end{array}$ & improvement \\
\hline $\begin{array}{l}\text { luminance at } 10 \mathrm{~V} \\
\left(\mathrm{~cd} \mathrm{\textrm {m } ^ { - 2 } ) ^ { a }}\right.\end{array}$ & $4750 \pm 470$ & $5940 \pm 610$ & $25.1 \%$ \\
\hline maximum EQE $(\%)^{a}$ & $1.19 \pm 0.12$ & $1.91 \pm 0.11$ & $60.5 \%$ \\
\hline $\operatorname{maximum} \eta_{\mathrm{L}}\left(\mathrm{cd} \mathrm{A}^{-1}\right)^{a}$ & $4.10 \pm 0.34$ & $6.18 \pm 0.50$ & $50.7 \%$ \\
\hline 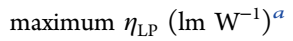 & $2.22 \pm 0.22$ & $3.69 \pm 0.39$ & $66.3 \%$ \\
\hline $\mathrm{LT}_{90}(\mathrm{~h})^{b}$ & 10.4 & $>20$ & $>92.3 \%$ \\
\hline
\end{tabular}

the $\beta$-phase in 90F8:10BT systematically boosts LED performance in terms of luminance, efficiencies (EQE, $\eta_{\mathrm{L}}$, and $\eta_{\mathrm{LP}}$ ), and operational stability $\left(\mathrm{LT}_{90}\right)$. In the following sections, we discuss, successively, the factors that contribute to the observed improvement in EQE, luminance, and operational stability.

EQE Enhancement. The LED EQE for fluorescent EML materials can be described as a product of terms that relate to the physical processes involved ${ }^{40}$

$$
\mathrm{EQE}=\eta_{\mathrm{CC}} \times \eta_{\mathrm{S} / \mathrm{T}} \times \mathrm{PLQE} \times \eta_{\text {out }}
$$

where (i) $\eta_{\mathrm{CC}}$ is the probability that injected holes and electrons Coulombically capture to form an exciton, (ii) $\eta_{\mathrm{S} / \mathrm{T}}$ is the ratio of singlet-to-triplet excitons that form, (iii) PLQE is the radiative probability for singlet exciton decay, and (iv) $\eta_{\text {out }}$ is the out-coupling efficiency, namely, the fraction of photons emitted within the device that escape to the exterior. For glassy and $\beta$-phase 90F8:10BT EMLs within an otherwise identical device structure, $\eta_{\mathrm{S} / \mathrm{T}}$ and $\eta_{\text {out }}$ are expected to be largely similar. Moreover, since their PLQEs were also found to be the same, the improvement in $\mathrm{EQE}$ most probably relates to the Coulomb capture probability $\eta_{\mathrm{CC}}$. An effective way to increase $\eta_{\mathrm{CC}}$ is to tune the position of the recombination zone, which ideally should be at the center of the EML or, in other words, away from the interfaces that favor nonradiative quenching of the excitons generated. This can be realized by balanced charge injection and transport, ideally through the achievement of ohmic contacts and comparably high hole and electron mobilities. To investigate this further, we measured the hole and electron mobilities in both the glassy and $\beta$-phase samples using two different methods, namely, (i) metal-insulatorsemiconductor structure-based charge carrier extraction by linearly increasing voltage (MIS-CELIV) and (ii) negative differential susceptance $(-\Delta B)$ measurements. The four (electron and hole for each method) unipolar diode structures used for these measurements are described in the Experimental Section below.

MIS-CELIV ${ }^{45}$ is a variant of the better known standard CELIV measurement. Hole and electron mobilities are determined by selectively injecting each into the semiconductor layer, following which they accumulate at the semiconductor-insulator interface. A linearly increasing voltage of the appropriate polarity is then applied to extract the injected carriers, resulting in separate transient current density traces for hole and electron transport. The key parameter used to calculate the mobility is $t_{2 j_{0}}$, which is the time for the output current density to rise to twice the displacement current density $j_{0}$. The mobility $\mu$ is then given by $^{46}$ 


$$
\mu=\frac{\pi^{2} d_{\mathrm{s}}^{2}}{8 A t_{2 \mathrm{j}_{0}}^{2}}\left(1+\frac{\varepsilon_{\mathrm{s}} d_{\mathrm{i}}}{\varepsilon_{\mathrm{i}} d_{\mathrm{s}}}\right)
$$

where $d_{\mathrm{s}}\left(d_{\mathrm{i}}\right)$ is the thickness of the semiconductor (insulator) layer, $\varepsilon_{\mathrm{s}}\left(\varepsilon_{\mathrm{i}}\right)$ is the permittivity of the semiconductor (insulator), and $A$ is the slope $\left(\mathrm{V} \mathrm{s}^{-1}\right)$ of the linearly increasing voltage.

Representative MIS-CELIV current density transients are shown in Figure S3a,b, and the mobility values extracted from the full set of measurements are presented in Figure 6a. Glassy samples have an electron mobility $\left(4.9 \times 10^{-7} \mathrm{~cm}^{2} \mathrm{~V}^{-1} \mathrm{~s}^{-1}\right)$ that is more than twice the hole mobility $\left(2.2 \times 10^{-7} \mathrm{~cm}^{2} \mathrm{~V}^{-1}\right.$ $\left.\mathrm{s}^{-1}\right)$. Generation of $\beta$-phase chain segments resolves this imbalance, yielding a more than 2-fold (118\%) mobility increase for holes to $4.7 \times 10^{-7} \mathrm{~cm}^{2} \mathrm{~V}^{-1} \mathrm{~s}^{-1}$, without significantly affecting the electron mobility other than by
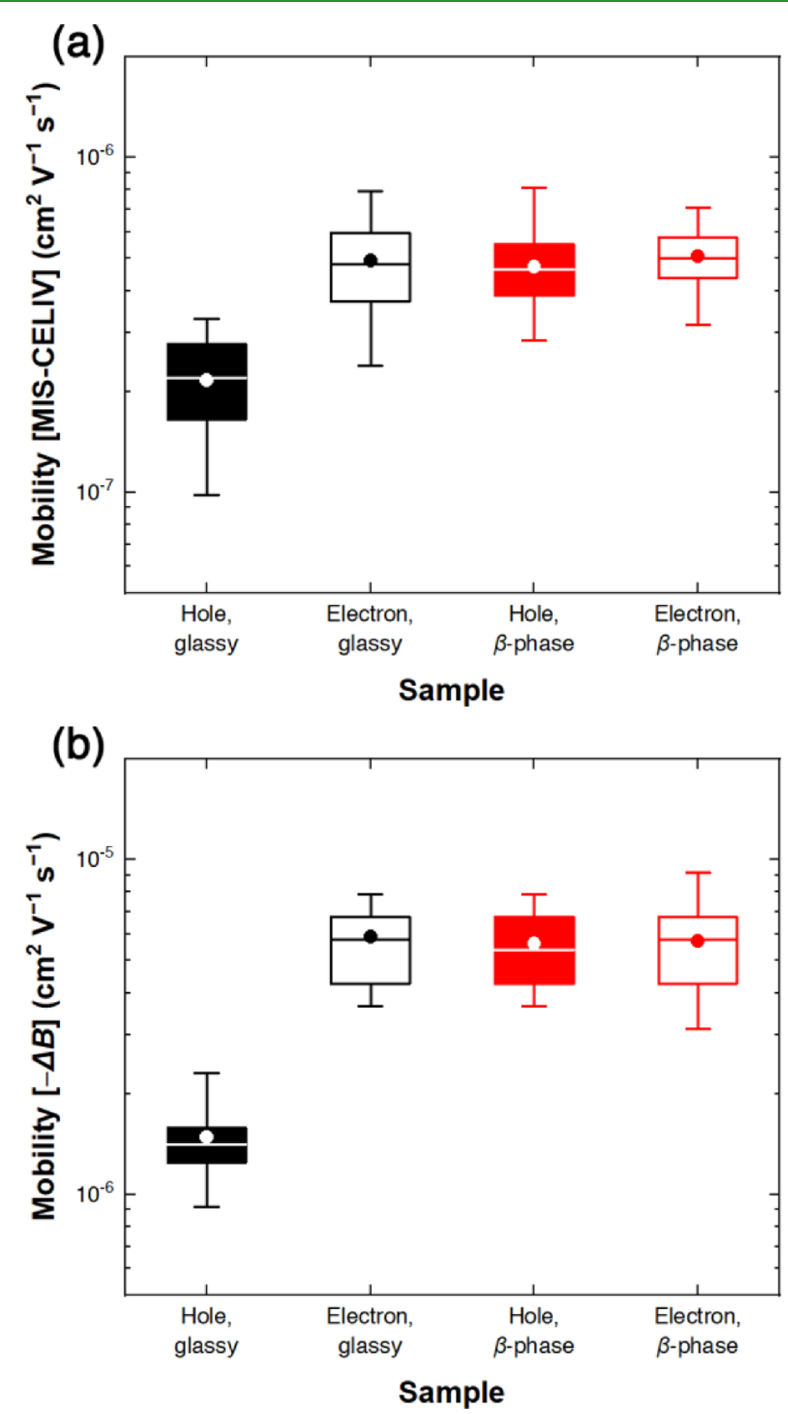

Figure 6. Hole and electron mobility values for the glassy and $\beta$-phase 90F8:10BT samples derived from (a) MIS-CELIV and (b) $-\Delta B$ measurements. Each measurement was performed for more than 10 samples, and the symbols display the minimum to maximum value range (vertical lines with end caps), the first and third quartiles (the bottom and top boundaries of the box, respectively), and the median and mean values (horizontal line and circle inside the box, respectively). desirably reducing its spread. $\beta$-phase-enhanced hole transport has already been observed in PFO, ${ }^{23,47}$ likely due to the longer conjugation length of $\beta$-phase chain segments, a more ordered and energetically favorable environment for hole transport, and a more interchain-connected microstructure. With $\beta$-phase chain segments present, hole and electron mobilities in 90F8:10BT become very closely matched, which is supportive of a favorable $\eta_{\mathrm{CC}}$.

The MIS-CELIV mobilities are relatively low compared to typical time-of-flight or dark-injection measurements on PFO and $\mathrm{F} \mathrm{BT}^{48,49}$ but not dissimilar to what is found for the copolymer 90F8:10BSP in which $10 \%$ of BSP moieties are included in an otherwise F8 backbone. ${ }^{30}$ Different measurements can yield different mobility values in the case that trapping effects occur and the measurements involve a large difference in charge carrier density, e.g., field-effect transistor mobility (high carrier density) versus space-charge-limited current or time-of-flight mobility measured in a diode (low carrier density) ${ }^{50}$ although there are also conjugated polymers for which the mobility is carrier density-independent. ${ }^{51}$

To check the reliability of the MIS-CELIV mobilities, we employed a second method, namely, negative differential susceptance $(-\Delta B)$ measurements. ${ }^{52}$ A small AC voltage with varying frequency is applied to a unipolar diode, superimposed on a DC bias voltage $V_{\mathrm{DC}}$ that ensures operation in the spacecharge-limited current regime. Details of the hole- and electron-only structures deployed for $-\Delta B$ measurements are provided in the Experimental Section below. The negative differential susceptance $-\Delta B$ varies with frequency $f$ as follows $^{53}$

$$
-\Delta B=2 \pi f\left(C-C_{\text {geo }}\right)
$$

where $C$ is the frequency-dependent capacitance and $C_{\text {geo }}$ is the geometric capacitance of the device. The obtained $-\Delta B$ vs $f$ curve is expected to exhibit a peak, which defines $f_{\mathrm{p}}$, from which the mobility can be calculated ${ }^{54}$

$$
\mu=\frac{d^{2} f_{\mathrm{p}}}{0.54\left(V_{\mathrm{DC}}-V_{\mathrm{bi}}\right)}
$$

where $d$ is the thickness of the $90 \mathrm{~F} 8: 10 \mathrm{BT}$ layer and $V_{\mathrm{bi}}$ is the built-in potential, approximated as the work function difference between the anode and cathode.

The $-\Delta B$-derived mobility values are shown in Figure $6 \mathrm{~b}$, and typical $-\Delta B$ vs $f$ curves are presented in Figure S3c. The same qualitative changes are observed, albeit with higher derived mobility values for $-\Delta B$ than for MIS-CELIV ( Table S1). Glassy 90F8:10BT has a hole mobility that is significantly lower than its electron mobility. Balanced charge transport then ensues following $\beta$-phase generation with the hole mobility increasing from $1.5 \times 10^{-6}$ to $5.6 \times 10^{-6} \mathrm{~cm}^{2} \mathrm{~V}^{-1}$ $\mathrm{s}^{-1}$ and the electron mobility marginally decreasing from $5.9 \times$ $10^{-6}$ to $5.7 \times 10^{-6} \mathrm{~cm}^{2} \mathrm{~V}^{-1} \mathrm{~s}^{-1}$.

Factors that may influence the difference between $-\Delta B$ and MIS-CELIV absolute mobility values are expected to relate to the assumptions that underpin the derivations. It has been previously shown that the MIS-CELIV method can lead to overestimation of the mobility when a metal and a semiconductor form an ohmic contact, whereas for an injection barrier larger than $\sim 0.47 \mathrm{eV}$, the mobility may be underestimated. $^{55}$ Here, the nominal energy levels (Figure $3 \mathrm{~b}$ ) would lead to an injection barrier of $0.5 \mathrm{eV}$, meaning that the obtained MIS-CELIV mobilities are likely to be slightly lower 
than the true values. Likewise, for the $-\Delta B$ measurement, the numerical factor 0.54 in eq 4 was derived in a case study of a poly ( $p$-phenylene vinylene) derivative. ${ }^{52,53}$ While this value has also been shown to be valid for different conjugated polymers, a deviation of \pm 0.1 is still possible when the charge transport is subject to different degrees of dispersion. ${ }^{56}$ If we assume that the charge transport is highly dispersive in 90F8:10BT films, i.e., the factor is larger than 0.54 , then the $-\Delta B$ mobilities presented here slightly overestimate the true values. This hypothesis is supported by dark-injection transient results for the 90F8:10BT samples (data not shown here), where no distinct maximum in the transient current density can be observed, indicating a high degree of charge carrier dispersion. ${ }^{57}$ This offers an interesting topic for future research.

The qualitative effect of $\beta$-phase chain segment formation that leads to balanced electron and hole mobility in these LEDs explains the observed improvement in EQE, with the resulting more centered recombination zone reducing interfacial quenching and any tendency to carrier leakage.

Luminance Enhancement. As noted above (and see Figure $5 b$ ), the turn-on voltage in $\beta$-phase LEDs is lower than that for the glassy devices, and $\beta$-phase LEDs yield higher luminance at a given voltage, from turn-on all the way up to 10 $\mathrm{V}$. The measured turn-on voltage comprises the built-in voltage $V_{\mathrm{bi}}$ required to equilibrate the Fermi levels of the anode and cathode and the additional voltage needed to overcome the barriers for hole and electron injection. ${ }^{40}$ Since the glassy and $\beta$-phase LEDs are identical other than their 90F8:10BT EML microstructure, $V_{\mathrm{bi}}$ should be the same for both. The lower turn-on voltage in $\beta$-phase LEDs thus indicates that one or both of the charge injection barriers must be lower. To further explore this behavior, current density $(J)$ and capacitance $(C)$ vs voltage $(V)$ measurements were performed on unipolar diodes (see the Experimental Section for structure and fabrication details) and LEDs, respectively.

Given that the $J-V$ curves (Figure S4) of electron-only glassy and $\beta$-phase diodes are almost identical, as also are their electron mobilities (Figure 6), we can conclude that the $\beta$ phase does not greatly affect electron injection properties. This is to be expected since the BT moieties provide the dominant contribution to the LUMO. ${ }^{38}$ In contrast, the hole current densities for the glassy and $\beta$-phase diodes show significantly different voltage dependences (Figure 7a). At low voltages, for both devices, $J \propto V$, as expected for ohmic transport associated with a background density of charge carriers within the film; for most conjugated polymers, as here, the background charge is p-type, potentially linked to oxidative doping by catalyst residues. The inverse film thickness and conductivity are then expected to determine the magnitude of $J$.

At higher voltages, above a threshold, the slope becomes much steeper, indicative of trap-mediated space-charge-limited transport with $J \propto V^{m}$. ${ }^{58}$ Here, $m=4.8$ for glassy and $m=4.5$ for $\beta$-phase devices. In the case of an exponential trap distribution, the lower value of $m$ would suggest a narrower trap state distribution for the $\beta$-phase 90F8:10BT, consistent with a higher degree of molecular order. However, in order to investigate the trap distribution in detail, a full temperature and film thickness dependence study would be required, ${ }^{58}$ which is beyond the scope of this work. The transition from ohmic to trap-mediated space-charge-limited current, estimated from the intercept of linear fits to the two regimes, is seen at $1.07 \pm 0.02$ and $0.39 \pm 0.01 \mathrm{~V}$ for the glassy and $\beta$-phase devices,

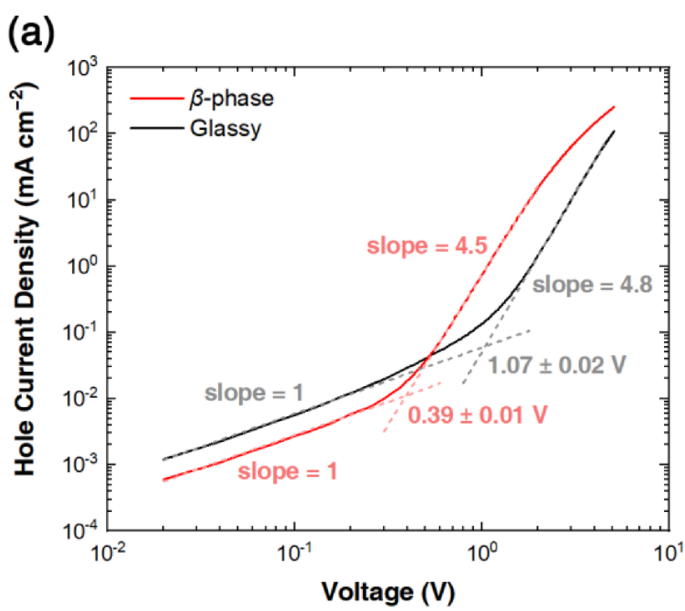

(b)

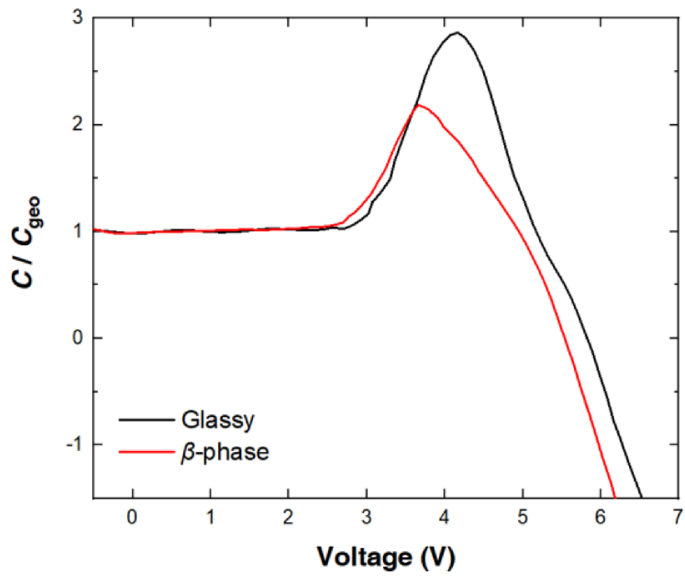

Figure 7. (a) $J-V$ characteristics of the hole-only glassy (black line) and $\beta$-phase (red line) 90F8:10BT diodes plotted on a double logarithmic scale. Linear fits (dashed lines) are shown to the ohmic and trap-mediated space-charge-limited current regimes for both microstructures. The transition voltage between these two regimes is estimated from the intercept of the linear fits. (b) $C-V$ curves for the glassy (black line) and $\beta$-phase (red line) 90F8:10BT LEDs.

respectively. The lower transition voltage in $\beta$-phase diodes confirms that their hole injection barriers must be lower. The transition is also significantly more abrupt for the $\beta$-phase diodes, again consistent with a narrower trap distribution. The lower injection barrier for the $\beta$-phase devices is further in line with prior results for PFO where $\beta$-phase formation was also seen to reduce the turn-on voltage. ${ }^{30}$ The chain extended $\beta$ phase conformation is expected to reduce the ionization potential (more delocalized $\pi$-electron density), , $19,25,59$ and calculations for fluorene octamers yield an $\sim 120$ to $140 \mathrm{meV}$ rise in the HOMO level. ${ }^{60}$ The optical gap is correspondingly $\sim 160 \mathrm{meV}$ smaller for the $\beta$-phase than for glassy PFO. ${ }^{61} \mathrm{An}$ additional reference point (upper bound) for the HOMO of $\beta$ phase chain segments is that they are deeper-lying than the cyclic-voltammetry-determined ionization potential of 95F8:5BSP, namely, $\sim 5.5 \mathrm{eV} ; \beta$-phase formation does not alter the turn-on voltage of 95F8:5BSP LEDs. ${ }^{30}$

The injection barriers were also investigated using $C-V$ measurements for glassy and $\beta$-phase LED structures. Figure $7 \mathrm{~b}$ shows a plot of the capacitance normalized to the geometric capacitance, $C_{\text {geo }}$ as a function of the applied voltage. While no significant charge carrier density is present within the device, the ratio $C / C_{\text {geo }}=1$. An increase in capacitance is then 
(a)

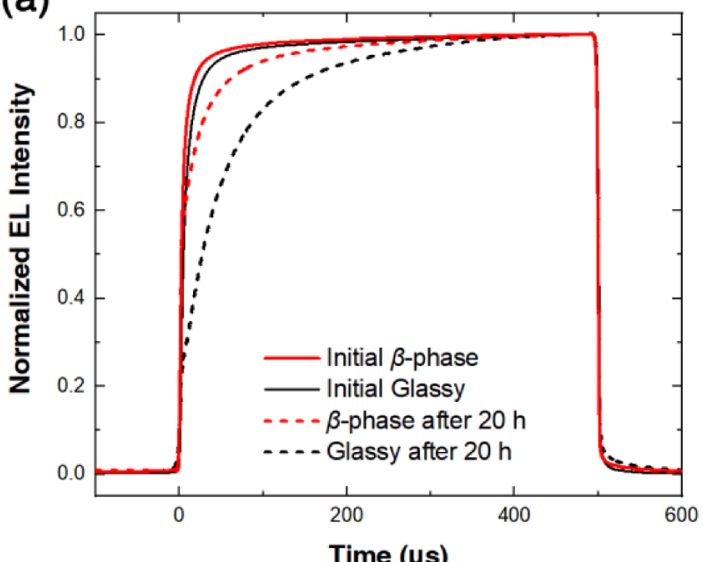

(c)

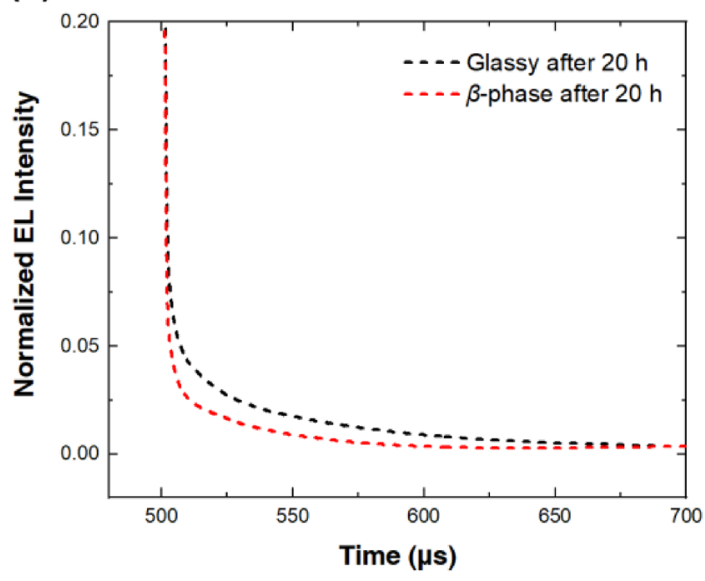

(b)

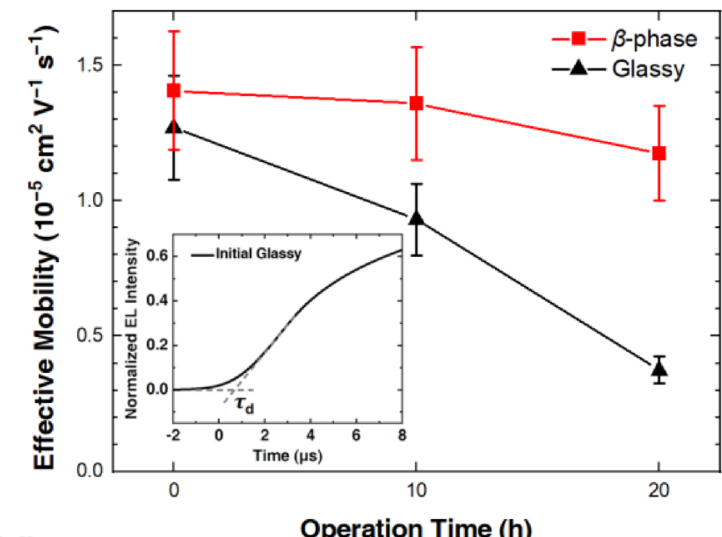

(d)

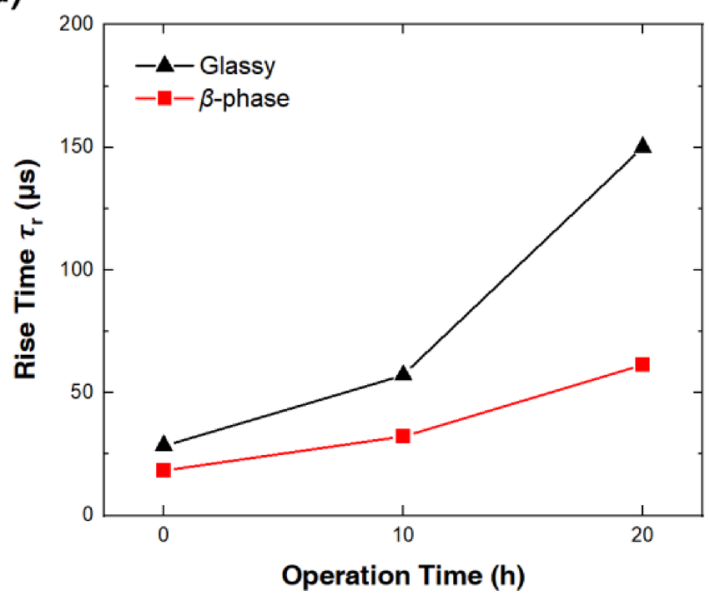

Figure 8. Transient EL curves and the deduced effective mobilities and rise times for 90F8:10BT LEDs during extended operation. (a) Transient EL curves for the glassy (black lines) and $\beta$-phase (red lines) LEDs before (solid lines) and after (dashed lines) 20 h of continuous operation at 80 $\mathrm{mA} \mathrm{cm}{ }^{-2}$, with starting luminance values of $\sim 2800$ and $\sim 3700 \mathrm{~cd} \mathrm{~m}^{-2}$ for the glassy and $\beta$-phase devices, respectively. (b) Deduced effective mobilities for the glassy (black triangle) and $\beta$-phase (red square) LEDs; the inset shows a typical data set, for a glassy device, used in the extraction of the delay time $\tau_{\mathrm{d}}$. (c) Enlarged turn-off response $(t>500 \mu \mathrm{s})$. (d) EL rise time values for the glassy (black triangle) and $\beta$-phase (red square) LEDs. The lines joining data points in panels (b) and (d) are guides to the eye. The transient EL curves after $10 \mathrm{~h}$ of operation are omitted for clarity.

observed from $\sim 2.7 \mathrm{~V}$ for both devices due to the injection of electrons and/or holes and their accumulation within the structure. From the energy level diagram (Figure 4b), hole injection is expected to have a $0.5 \mathrm{eV}$ barrier height while the $\mathrm{LiF} / \mathrm{Al}$ cathode with a work function of $\sim 2.75 \mathrm{eV}$ should form an ohmic contact to $90 \mathrm{~F} 8: 10 \mathrm{BT}^{42}$ It is, therefore, expected that electrons will be injected first (at $2.7 \mathrm{~V}$ ), with very similar behavior for both 90F8:10BT microstructures, as is indeed seen. At higher voltage, holes will also be able to surmount their associated barrier and be injected, yielding charge carrier combination to form exciton states and a fall in capacitance. The voltage, $V_{\mathrm{p}}$, at peak capacitance has previously been shown by numerical simulation to be a measure of the second injection barrier. ${ }^{62}$ In Figure $7 \mathrm{~b}$, the $V_{\mathrm{p}}$ values for the glassy and $\beta$-phase devices are $4.2 \pm 0.1$ and $3.7 \pm 0.1 \mathrm{~V}$, respectively, confirming again that the hole injection barrier is reduced by $\beta$ phase chain segment formation.

The sensitivity of hole-related and contrasting insensitivity of electron-related injection and transport to $\beta$-phase formation can be understood from previous quantum chemical calculations. Winfield et al. $^{38}$ have shown that for F8-BT oligomers with a BT ratio varying from 8.3 to $50 \%$, the LUMO is always highly localized on the BT moieties, whereas the
HOMO delocalizes to the degree allowed by the chain geometry. Torsional twists limit wave function spread, and therefore, during $\beta$-phase formation where the change in conformation is to a chain extended planar structure, the $\mathrm{HOMO}$ rises in energy and the hole injection barrier lowers. In contrast, the BT-dominated LUMO is barely affected by the change in conformation, leaving the electron injection barrier largely unchanged. In addition, Cornil et al. ${ }^{59}$ have calculated the geometry-dependent interchain transfer integrals, critical to mobility in the hopping transport regime. In F8BT (alternating copolymer) when two neighboring polymer chains translate relative to each other along their long-chain axis, it was found that the transfer integral for hole transport undulates as a function of the degree of translation while that for electron transport is fairly constant when the BT units on one chain face the F8 units on the other. Here, only $10 \%$ of the chain is composed of BT units, but a cautious generalization should still be possible. This would suggest that adoption of the $\beta$ phase should alter the transfer integral for hole transport and thus the hole mobility. However, the low BT content makes it highly likely that the BT units on one polymer chain will face the F8 units on neighboring chains, making the transfer 
integral for electron transport rather insensitive to conformation change.

The reduced hole injection barrier and more balanced charge transport engendered by $\beta$-phase chain segment formation then lead to an increased exciton formation rate and explain the higher luminance that is observed. One question that arises here is whether the dipping process leads to any significant changes in the interface between the TFB and 90F8:10BT layers due to intermixing. We note, however, in this context that the TFB layer was cross-linked at $180{ }^{\circ} \mathrm{C}$ for $1 \mathrm{~h}$ before the 90F8:10BT films were spin-coated on top. In a previous paper, ${ }^{63}$ it was shown that after the cross-linking treatment used here, the TFB layer is highly solvent-resistant, so significant mixing is not expected. Furthermore, the same spin-coating parameters were used for both the films that subsequently remained in the glassy state and those that were dipped to generate the $\beta$-phase. Any intermixing at the interface might be expected to more readily occur during this step, given the solvents used, than during the dipping step and thus to be the same for both device types (glassy and $\beta$-phase). Finally, intermixing is even less likely to occur exclusively in the presence of the poor solvent (cyclohexane/isopropanol mixture) used for dipping.

Operational Stability Enhancement. Transient electroluminescence (EL) measurements were performed on glassy and $\beta$-phase LEDs to investigate the operational stability differences shown in Figure 5e. The devices were tested under constant current density $\left(80 \mathrm{~mA} \mathrm{~cm}^{-2}\right)$ operation at $t=0,10$, and $20 \mathrm{~h}$. Typical transient EL curves comprise four features (Figure 8a): (i) a delayed onset of EL intensity following application of the voltage pulse, with characteristic time $\tau_{\mathrm{d}}$ (Figure $8 \mathrm{~b}$ inset). This delay time, $\tau_{\mathrm{d}}$, can be used to extract an effective mobility $\mu_{\text {eff }}$ which combines contributions from the hole and electron mobilities ${ }^{64}$

$$
\mu_{\mathrm{eff}}=\frac{d^{2}}{\tau_{\mathrm{d}}\left(V-V_{\mathrm{bi}}\right)}
$$

where $V_{\mathrm{bi}}$ is the built-in voltage and, assuming that the charges transit the whole device (i.e., none are trapped within it), $d$ is the thickness of the active layer; (ii) the rise in EL intensity to its steady-state value, with characteristic time $\tau_{\mathrm{r}}$ to reach $90 \%$ of maximum luminance. A longer $\tau_{\mathrm{r}}$ signals lower mobility and/or higher trap density; (iii) steady-state EL while the voltage pulse is on; and (iv) the EL decay after turning the applied voltage off. During the decay, oppositely charged carriers can de-trap and combine to yield delayed EL emission. Slow decays therefore indicate higher trap densities. The first and fourth features, respectively, reflect carrier mobilities and trap densities, while the second is representative of both.

Figure $8 \mathrm{~b}$ shows the effective mobility values extracted from $\tau_{\mathrm{d}}$. The initial $\beta$-phase measurement yields a higher value than for the glassy device, consistent with the results from MISCELIV and $-\Delta B$ measurements. During continuous operation, the effective mobility for both devices reduces and the difference between them increases, especially after $10 \mathrm{~h}$, signaling a significant degradation in charge transport for the glassy LEDs. After $20 \mathrm{~h}$ of operation, the effective mobility in $\beta$-phase devices drops to $1.17 \times 10^{-5} \mathrm{~cm}^{2} \mathrm{~V}^{-1} \mathrm{~s}^{-1}$, i.e., $84 \%$ of its initial value, but for the glassy devices, only $29 \%$ of the initial effective mobility, $3.74 \times 10^{-6} \mathrm{~cm}^{2} \mathrm{~V}^{-1} \mathrm{~s}^{-1}$, remains. The higher effective mobility for $\beta$-phase LEDs after extended operation, relative to glassy devices, was also confirmed by additional $-\Delta B$ measurements on LED structures before and after continuous aging (Figure S5).

The EL decay curves after $20 \mathrm{~h}$ of operation, shown on an expanded intensity scale in Figure 8c, have a correspondingly slower drop-off in luminance for glassy devices, suggesting that a higher trap density is present. This is further confirmed by a sharp increase of the low-frequency capacitance for glassy LEDs after $20 \mathrm{~h}$ of operation (Figure S6a) and a moderate increase for $\beta$-phase devices (Figure S6b). ${ }^{65}$ Such traps are expected to contribute to the lower effective mobility. The origin of the traps in this case remains unknown, but a variety of chemical and physical changes are known to contribute. ${ }^{66,67}$ Influenced by both charge carrier mobility and trapping, the deduced rise times, $\tau_{\mathrm{r}}$, shown in Figure $8 \mathrm{~d}$ follow the trends reported above, namely, that $\beta$-phase devices are less prone to forms of degradation that lead to increased trap densities and/ or other negative impacts on mobility. In this context, we also note that during the operation of an LED, the shorter exciton radiative lifetime following $\beta$-phase generation (Figure 3) means that there is less chance to interact with charge carriers and each other, potentially helping to reduce trap formation.

As a final characterization, the EL spectra for glassy and $\beta$ phase LEDs were recorded before and after $20 \mathrm{~h}$ of continuous operation (Figure 9). The change in EL spectra is rather

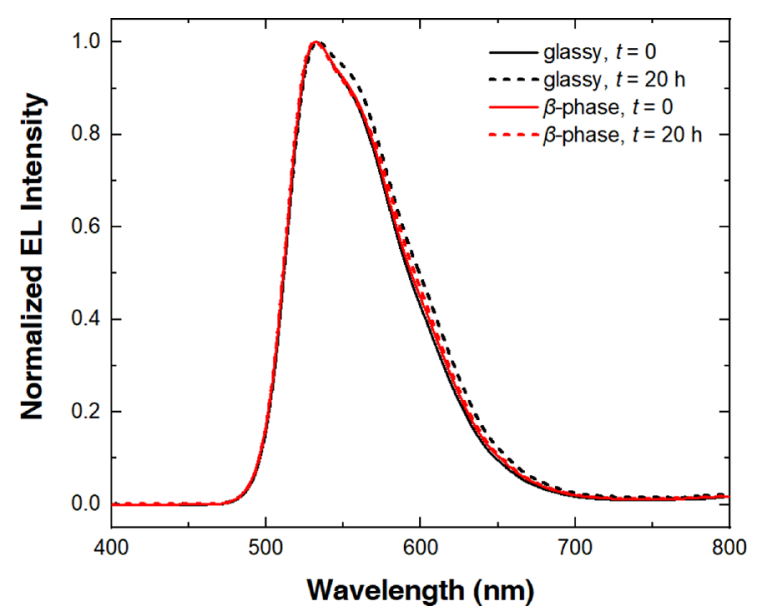

Figure 9. Changes in EL spectra for the 90F8:10BT glassy (black) and $\beta$-phase (red) LEDs following $20 \mathrm{~h}$ of continuous operation at 80 $\mathrm{mA} \mathrm{cm}{ }^{-2}$. Initial spectra are shown by solid lines, and those following $20 \mathrm{~h}$ of operation are shown by dashed lines.

modest with a slight broadening of the peak on its red edge, while the short wavelength side is essentially unchanged. The changes are more noticeable, but certainly not pronounced, for glassy devices with a slight increase in relative emission strength at $\sim 560 \mathrm{~nm}$. The relative weighting of F8BT spectral components has been reported to depend on interchain, interBT unit packing, with an increase in direct BT-to-BT contacts between chains increasing the strength of the longer wavelength shoulder. ${ }^{68}$ More generally, aggregation effects often yield red-shifted and broadened PL and EL spectra, and certain chemical species formed during photo-oxidation of fluorene units can also contribute, with the resulting fluorenone excimer emission at $\sim 535 \mathrm{~nm} .{ }^{69}$ A more detailed study of the chemical stability of $\beta$-phase $90 \mathrm{~F} 8: 10 \mathrm{BT}$ would be an interesting topic for future research. 


\section{SUMMARY AND CONCLUSIONS}

The research reported in this study demonstrates a successful generalization of the use of chain conformation to enhance the device performance of conjugated polymer LEDs with respect to both their efficiency and stability. Generation of the $\beta$-phase conformation for PFO has been shown previously to enhance the performance of LEDs fabricated therefrom. Here, we use the generation of $\beta$-phase chain segments in a commercially available $10 \%$ BT-containing PFO-based copolymer, 90F8:10BT, to yield significantly improved LED efficiency and stability. A detailed investigation of device-related material properties and the performance of LEDs with and without the $\beta$-phase allows an understanding of the role that this conformation change plays.

The absorption spectra of 90F8:10BT combine the spectral features of $\mathrm{PFO}$ and alternating copolymer F8BT, and generation of $\beta$-phase chain segments via SVA or dipping yields the characteristic $\beta$-phase peak at $\sim 430 \mathrm{~nm}$. Spectral deconvolution allows estimation of the $\beta$-phase content, typically in the range of $5-10 \%$. Dipping is the preferred method for device fabrication and gives an $\sim 5 \% \beta$-phase component. Unlike absorption spectra, the luminescence emission is dominated by the green-yellow BT-localized exciton with residual F8-related blue emission having a steadystate peak intensity $\leq 0.1 \%$ of the green-yellow emission. Efficient and rapid energy transfer occurs from F8-localized excitons, and the emission then proceeds with a single exponential decay with a time constant that is very close to but slightly longer than that of F8BT $(\sim 2.0 \mathrm{~ns})$ : the $\beta$-phase has a decay time of $\sim 2.3 \mathrm{~ns}$, compared with $\sim 2.5 \mathrm{~ns}$ for glassy 90F8:10BT. The emission efficiency is also high $(\sim 80 \%)$ and independent of the $\beta$-phase fraction (for the range studied). The shorter decay time with the same PLQE implies a higher oscillator strength for the more ordered $\beta$-phase films.

A detailed investigation (using MIS-CELIV, $-\Delta B, C-V, J-$ $V$, and transient EL measurements) of the charge injection, transport, and trapping, and LED electrical and optical characteristics for 90F8:10BT shows that glassy films give rise to imbalanced charge carrier injection and transport with significantly lower hole than electron mobility and higher LED turn-on voltage. With the $\beta$-phase, the hole mobility rises to become close to the electron mobility and the injection barrier is reduced. This is evident from the MIS-CELIV, $-\Delta B$, and $C-V$ measurements. $\beta$-phase 90F8:10BT LEDs consequently yield higher luminance and have higher $\eta_{\mathrm{L}}, \eta_{\mathrm{LP}}$, and $\mathrm{EQE}$ values. Both glassy and $\beta$-phase show the effects of trapping, but they are more pronounced for the glassy phase of 90F8:10BT. This is particularly clear from the $J-V$ and transient EL measurements.

$\beta$-phase formation significantly enhances LED stability relative to glassy 90F8:10BT devices under continuous operation at $80 \mathrm{~mA} \mathrm{~cm}{ }^{-2}$. The glassy devices show strongly increased carrier trapping effects leading to reduced transient EL effective mobilities, longer transient EL rise and turn-off times, and enhanced device capacitance at low frequency. The corresponding performance changes for $\beta$-phase devices are significantly weaker, such that, for example, the average EL $\mathrm{LT}_{90}$ value is more than double the $\sim 10 \mathrm{~h}$ found for glassy devices, despite the starting luminance values at $80 \mathrm{~mA} \mathrm{~cm} \mathrm{~cm}^{-2}$ being $\sim 3700 \mathrm{~cd} \mathrm{~m}^{-2}$ for $\beta$-phase and $\sim 2800 \mathrm{~cd} \mathrm{~m}^{-2}$ for glassy. The detailed nature of the traps and how the $\beta$-phase formation affects their appearance and/or activity remain a topic for future study. EL spectra show relatively little and not very distinct change, with a red-edge broadening that is larger for the glassy phase but not major in either case. No equivalent of the green-band emission that downgrades the blue emission color of PFO LEDs is observed here, albeit that if it appeared in the same spectral position as for PFO, it would strongly overlap with the observed undegraded 90F8:10BT emission spectrum.

90F8:10BT has been proven to be an interesting test case with which to study the influence of conformation on the fundamental and applied properties of fluorene-based conjugated copolymer emission materials. It provides a system within which charge carrier injection, transport, and exciton characteristics can be tuned as a function of the physical structure. This broadens the applicability range of conformation-imparted improvements to LED performance, an intervention level that lies between chemical modification and device engineering.

\section{EXPERIMENTAL SECTION}

Materials. 90F8:10BT $\left(M_{\mathrm{w}}=55,000\right.$, polydispersity $n=3.6$, product name ADS233YE) was purchased from American Dye Source and used as received. Toluene (99.8\%), cyclohexane (99.5\%), isopropanol $(99.5 \%)$, and TFB $\left(M_{\mathrm{w}}>30,000\right)$ were supplied by Sigma-Aldrich. PEDOT:PSS (Clevios PVP AI 4083) was provided by Heraeus. Evaporation materials (99.9\% LiF and $99.99 \% \mathrm{Al}$ ) were purchased from Kurt J. Lesker. Prepatterned ITO substrates $(10 \Omega$ / square) were sourced from Thin Film Devices. All chemicals were used as received without further purification.

$\boldsymbol{\beta}$-Phase Generation. The $\beta$-phase was generated within 90F8:10BT thin films spin-coated on fused silica substrates using two methods, namely, SVA and dipping. For the former, the sample was placed together with $\sim 5 \mathrm{~mL}$ of toluene in a vial (to induce a saturated toluene vapor) within a sealed container and kept there at room temperature for $24 \mathrm{~h}$ in the dark. For the dipping method, the substrate-coated thin film was immersed in $\sim 5 \mathrm{~mL}$ of cyclohexane/ isopropanol mixture (volume ratio $=1: 1$ ) for $90 \mathrm{~s}$ and then dried under flowing nitrogen. The sample remained intact during dipping, but its thickness reduced, suggesting partial dissolution. Taking this into account, to achieve comparable film thickness samples, the starting films for dipping were deliberately fabricated thicker by adjusting the spin-coating speed in order that the final thickness would be the same as for the glassy and SVA samples.

LED Fabrication and Characterization. The typical LED structure used in this study was ITO/PEDOT:PSS $(30 \mathrm{~nm}) / \mathrm{TFB}$ $(15 \mathrm{~nm}) / 90 \mathrm{~F} 8: 10 \mathrm{BT}(80 \mathrm{~nm}) / \mathrm{LiF}(1 \mathrm{~nm}) / \mathrm{Al}(100 \mathrm{~nm})$. To start with, the prepatterned ITO-coated glass substrates were cleaned in ultrasonic baths with acetone and isopropanol for $10 \mathrm{~min}$ each followed by UV-ozone treatment for $10 \mathrm{~min}$. Next, the Clevios PVP AI 4083 PEDOT:PSS aqueous solution was spin-coated on top of the ITO substrates at $2500 \mathrm{rpm}$ for $45 \mathrm{~s}$ and then annealed at $150{ }^{\circ} \mathrm{C}$ for $15 \mathrm{~min}$ in air to remove residual moisture. These structures were transferred to a nitrogen-filled glovebox for polymer layer deposition. TFB was spin-coated at $1000 \mathrm{rpm}$ for $45 \mathrm{~s}$ from $2 \mathrm{mg} \mathrm{mL}^{-1}$ toluene solution and then annealed at $180{ }^{\circ} \mathrm{C}$ for $60 \mathrm{~min}$ to enable partial cross-linking, ${ }^{63}$ thus preventing damage from the same toluene solvent used to deposit the 90F8:10BT film on top. This film was spin-coated at 1000 (for glassy samples) or $770 \mathrm{rpm}$ (for $\beta$-phase samples) for $45 \mathrm{~s}$ from $10 \mathrm{mg} \mathrm{mL}^{-1}$ toluene solution followed by thermal annealing at $80{ }^{\circ} \mathrm{C}$ for $10 \mathrm{~min}$. The dipping method was found to be preferable for generating $\beta$-phase chain segments in device samples. The LED fabrication was completed by transferring the 90F8:10BT-coated samples to a thermal evaporator where $\mathrm{LiF}$ $\left(0.2 \AA^{-1}\right)$ and $\mathrm{Al}\left(1 \AA \mathrm{s}^{-1}\right)$ were deposited to form the top cathode. All LED samples were encapsulated using UV-cured epoxy adhesive (Lumtec LT-U001) and a glass coverslip before being brought out of the glovebox. 
LEDs were characterized using a custom-built setup, consisting of a Keithley 2636 sourcemeter, an Ocean Optics Maya2000 Pro spectrometer, and a Newport Oriel Instruments 70682NS integrating sphere. Controlled by bespoke software, the setup can apply a voltage to a given pixel and simultaneously record current density, luminance, EQE, luminous efficiency, and luminous power efficiency. The accelerated device lifetime test was also carried out using this setup. The steady-state and transient EL measurements were performed using a Fluxim PAIOS measurement platform operated via Characterization Suite 4.2 software. The former was carried out at $10 \mathrm{~V}$, while a $10 \mathrm{~V}, 500 \mu$ s voltage pulse was used in the latter case. Reported LED device performance values are averages for multiple device structures ( $>20$ in every case and 30 in some cases).

Optical Property Measurements. 90F8:10BT thin films deposited on fused silica substrates, with the same spin-coating and post-treatment details as used for the LED device EMLs, were used for optical spectroscopy. Absorption $(A)$ spectra were extracted from transmittance $(T)$ and reflectance $(R)$ spectra using $A(\lambda)=1-T(\lambda)$ $-R(\lambda)$, with $T(\lambda)$ and $R(\lambda)$ measured using a PerkinElmer Lambda $1050 \mathrm{UV}-$ vis-NIR spectrometer with a $100 \mathrm{~mm}$ integrating sphere accessory. The $\beta$-phase fractions were calculated from the difference in absorption spectra between the glassy and $\beta$-phase samples, following the method described in ref 21. Steady-state PL spectra (excitation wavelength $=380 \mathrm{~nm}$ ) were obtained using a Horiba FluoroMax-4 spectrofluorometer with a Quanta- $\varphi$ F-3029 integrating sphere. The PLQEs were calculated following ref 21. Transient PL measurements were performed using a time-correlated single-photon counting (TCSPC) setup (FluoTime 300 PicoQuant GmbH). Samples were photoexcited by a $398 \mathrm{~nm}$ picosecond pulsed diode laser (LDH-D-C-405 M, PicoQuant GmbH) with a PDL 820 driver at a $5 \mathrm{MHz}$ repetition rate, with a pulse duration of 16 ps. The PL emission was collected with a lens and coupled into a grating spectrometer (SP-2558, Princeton Instruments) equipped with a photon-counting detector (PDM series, Micro Photon Devices), with the system controlled electronically using a TCSPC event timer (PicoHarp 300, PicoQuant GmbH). Measurements were carried out in ambient air.

Unipolar Diode Structures for Electrical Property Measurements. Samples for MIS-CELIV measurements were fabricated with the structures ITO/PEDOT:PSS/90F8:10BT/ $\mathrm{MgF}_{2}(10 \mathrm{~nm}) / \mathrm{Al}$ for hole mobility and ITO/MgF $2(10 \mathrm{~nm}) / 90 \mathrm{~F} 8: 10 \mathrm{BT} / \mathrm{LiF} / \mathrm{Al}$ for electron mobility measurements. For $-\Delta B$ measurements, the test samples were either an ITO/PEDOT:PSS/90F8:10BT/ $\mathrm{MoO}_{3}(10$ $\mathrm{nm}) / \mathrm{Al}$ hole-only device or an ITO/ZnO $(30 \mathrm{~nm}) / 90 \mathrm{~F} 8: 10 \mathrm{BT} / \mathrm{LiF} /$ Al electron-only device. Here, $\mathrm{MgF}_{2}$ and $\mathrm{MoO}_{3}$ were thermally evaporated (both at $0.2 \AA \mathrm{s}^{-1}$ ), while $\mathrm{ZnO}$ was solution-processed. All other layers were fabricated following the same protocols as in LED devices. MIS-CELIV ( $V_{\text {offset }}=8 \mathrm{~V}, A=200-1000$ with $200 \mathrm{~V} \mathrm{~ms}^{-1}$ steps) and $-\Delta B\left(V_{\mathrm{AC}}=0.05 \mathrm{~V}, V_{\mathrm{DC}}=5-7 \mathrm{~V}\right.$ with $1 \mathrm{~V}$ steps $)$ mobility, as well as the $C-V\left(V_{\mathrm{AC}}=0.05 \mathrm{~V}, f=1000 \mathrm{~Hz}\right)$ and $C-f$ $\left(V_{\mathrm{AC}}=0.05 \mathrm{~V}, V_{\mathrm{DC}}=0\right)$ measurements for LED devices, were performed using the Fluxim PAIOS measurement platform with Characterization Suite 4.2 software. The $J-V$ characteristics for unipolar devices, with the same sample structure used for $-\Delta B$ measurements, were obtained using a Keithley 2400 sourcemeter.

Thin-Film Characterization. The film thickness was measured using a Veeco Dektak 150 profilometer. Film morphology and roughness were characterized using a Nanosurf CoreAFM atomic force microscope.

\section{ASSOCIATED CONTENT}

\section{(s) Supporting Information}

The Supporting Information is available free of charge at https://pubs.acs.org/doi/10.1021/acsami.0c18490.

Absorption spectral deconvolution of $\beta$-phase samples; AFM images of glassy and $\beta$-phase samples; typical mobility measurement results using MIS-CELIV and $-\Delta B$ methods and mobility summary; $J-V$ character- istics of electron-only devices; effective mobility measurement results using $-\Delta B$ methods on initial and aged LEDs; $C-f$ characteristics for initial and aged LEDs (PDF)

\section{AUTHOR INFORMATION}

\section{Corresponding Author}

Donal D. C. Bradley - Physical Science and Engineering Division, King Abdullah University of Science and Technology (KAUST), Thuwal 23955-6900, Saudi Arabia; Clarendon Laboratory, Department of Physics, University of Oxford, Oxford OX1 3PU, UK; (1) orcid.org/0000-0001-87135060; Email: donal.bradley@kaust.edu.sa

\section{Authors}

Bingjun Wang - Clarendon Laboratory, Department of Physics, University of Oxford, Oxford OX1 3PU, UK; - orcid.org/0000-0001-5832-4081

Hao Ye - Clarendon Laboratory, Department of Physics, University of Oxford, Oxford OX1 3PU, UK

Moritz Riede - Clarendon Laboratory, Department of Physics, University of Oxford, Oxford OX1 3PU, UK; 10 orcid.org/ 0000-0002-5399-5510

Complete contact information is available at: https://pubs.acs.org/10.1021/acsami.0c18490

\section{Notes}

The authors declare no competing financial interest.

\section{ACKNOWLEDGMENTS}

B.W. and D.D.C.B. thank the China Scholarship Council (CSC, No. 201700260029), Pacific Alliance Group (PAG), China Oxford Scholarship Fund (COSF), and King Abdullah University of Science and Technology (KAUST, No. 121053645) for studentship support. H.Y. and D.D.C.B. thank the Jiangsu Industrial Technology Research Institute (JITRI) and the JITRI-Oxford IMPACT Institute for project funding (R57149/CN001). The authors further thank Professors Henry Snaith and Stephen Morris for access to facilities and Drs. Chen Sun and Pascal Kaienburg for fruitful discussions.

\section{REFERENCES}

(1) Burroughes, J. H.; Bradley, D. D. C.; Brown, A. R.; Marks, R. N.; Mackay, K.; Friend, R. H.; Burns, P. L.; Holmes, A. B. Light-Emitting Diodes Based on Conjugated Polymers. Nature 1990, 347, 539-541.

(2) Perevedentsev, A.; Stavrinou, P. N.; Smith, P.; Bradley, D. D. C. Solution-Crystallization and Related Phenomena in 9,9-DialkylFluorene Polymers. II. Influence of Side-Chain Structure. J. Polym. Sci., Part B: Polym. Phys. 2015, 53, 1492-1506.

(3) Grell, M.; Bradley, D. D. C.; Inbasekaran, M.; Woo, E. P. A Glass-Forming Conjugated Main-Chain Liquid Crystal Polymer for Polarized Electroluminescence Applications. Adv. Mater. 1997, 9, 798-802.

(4) Grell, M.; Bradley, D. D. C.; Long, X.; Chamberlain, T.; Inbasekaran, M.; Woo, E. P.; Soliman, M. Chain Geometry, Solution Aggregation and Enhanced Dichroism in the Liquidcrystalline Conjugated Polymer Poly(9,9-dioctylfluorene). Acta Polym. 1998, 49, 439-444.

(5) Ariu, M.; Lidzey, D. G.; Sims, M.; Cadby, A. J.; Lane, P. A.; Bradley, D. D. C. The Effect of Morphology on the TemperatureDependent Photoluminescence Quantum Efficiency of the Conjugated Polymer Poly(9, 9-dioctylfluorene). J. Phys.: Condens. Matter 2002, 14, 9975-9986. 
(6) Redecker, M.; Bradley, D. D. C.; Inbasekaran, M.; Woo, E. P. Mobility Enhancement Through Homogeneous Nematic Alignment of a Liquid-Crystalline Polyfluorene. Appl. Phys. Lett. 1999, 74, 14001402.

(7) Whitehead, K. S.; Grell, M.; Bradley, D. D. C.; Jandke, M.; Strohriegl, P. Highly Polarized Blue Electroluminescence from Homogeneously Aligned Films of Poly(9,9-dioctylfluorene). Appl. Phys. Lett. 2000, 76, 2946-2948.

(8) Stavrinou, P. N.; Ryu, G.; Campoy-Quiles, M.; Bradley, D. D. C. The Change in Refractive Index of Poly(9,9-dioctylfluorene) due to the Adoption of the $\beta$-phase Chain Conformation. J. Phys.: Condens. Matter 2007, 19, 466107.

(9) Le Roux, F.; Bradley, D. D. C. Conformational Control of Exciton-Polariton Physics in Metal-Poly(9,9-dioctylfluorene)-Metal Cavities. Phys. Rev. B 2018, 98, 195306.

(10) Le Roux, F.; Taylor, R. A.; Bradley, D. D. C. Enhanced and Polarization-Dependent Coupling for Photoaligned Liquid Crystalline Conjugated Polymer Microcavities. ACS Photonics 2020, 7, 746-758.

(11) Grice, A. W.; Bradley, D. D. C.; Bernius, M. T.; Inbasekaran, M.; Wu, W. W.; Woo, E. P. High Brightness and Efficiency Blue Light-Emitting Polymer Diodes. Appl. Phys. Lett. 1998, 73, 629-631.

(12) Neher, D. Polyfluorene Homopolymers: Conjugated LiquidCrystalline Polymers for Bright Blue Emission and Polarized Electroluminescence. Macromol. Rapid Commun. 2001, 22, 13651385.

(13) Bradley, D. D. C.; Grell, M.; Long, X.; Mellor, H.; Grice, A. W.; Inbasekaran, M.; Woo, E. P. Influence of Aggregation on the Optical Properties of a Polyfluorene. Proc. SPIE-Int. Soc. Opt. Eng. 1997, 3145, 254-259.

(14) Chunwaschirasiri, W.; Tanto, B.; Huber, D. L.; Winokur, M. J. Chain Conformations and Photoluminescence of Poly(di- $n$-octylfluorene). Phys. Rev. Lett. 2005, 94, 107402.

(15) Khan, A. L. T.; Sreearunothai, P.; Herz, L. M.; Banach, M. J.; Köhler, A. Morphology-Dependent Energy Transfer within Polyfluorene Thin Films. Phys. Rev. B 2004, 69, No. 085201.

(16) Peet, J.; Brocker, E.; Xu, Y.; Bazan, G. C. Controlled $\beta$-Phase Formation in Poly(9,9-di-n-octylfluorene) by Processing with Alkyl Additives. Adv. Mater. 2008, 20, 1882-1885.

(17) Zhang, Q.; Chi, L.; Hai, G.; Fang, Y.; Li, X.; Xia, R.; Huang, W.; $\mathrm{Gu}, \mathrm{E}$. An Easy Approach to Control $\beta$-Phase Formation in PFO Films for Optimized Emission Properties. Molecules 2017, 22, 315.

(18) Worsfold, O.; Hill, J.; Heriot, S. Y.; Fox, A. M.; Bradley, D. D. C.; Richardson, T. H. Langmuir and Langmuir-Blodgett (LB) Film Properties of Poly(9,9-dioctylfluorene). Mater. Sci. Eng., C 2003, 23, $541-544$.

(19) Cadby, A. J.; Lane, P. A.; Mellor, H.; Martin, S. J.; Grell, M.; Giebeler, C.; Bradley, D. D. C.; Wohlgenannt, M.; An, C.; Vardeny, Z. V. Film Morphology and Photophysics of Polyfluorene. Phys. Rev. B 2000, 62, 15604-15609.

(20) Ryu, G.; Stavrinou, P. N.; Bradley, D. D. C. Spatial Patterning of the $\beta$-Phase in Poly(9,9-dioctylfluorene): A Metamaterials-Inspired Molecular Conformation Approach to the Fabrication of Polymer Semiconductor Optical Structures. Adv. Funct. Mater. 2009, 19, 3237-3242.

(21) Perevedentsev, A.; Chander, N.; Kim, J.-S.; Bradley, D. D. C. Spectroscopic Properties of Poly(9,9-dioctylfluorene) Thin Films Possessing Varied Fractions of $\beta$-Phase Chain Segments: Enhanced Photoluminescence Efficiency via Conformation Structuring. J. Polym. Sci., Part B: Polym. Phys. 2016, 54, 1995-2006.

(22) Perevedentsev, A.; Sonnefraud, Y.; Belton, C. R.; Sharma, S.; Cass, A. E. G.; Maier, S. A.; Kim, J.-S.; Stavrinou, P. N.; Bradley, D. D. C. Dip-pen Patterning of Poly(9,9-dioctylfluorene) Chain-Conformation-Based Nano-Photonic Elements. Nat. Commun. 2015, 6, 5977.

(23) Lu, H. H.; Liu, C. Y.; Chang, C. H.; Chen, S. A. Self-Dopant Formation in Poly(9,9-di-n-octylfluorene) Via a Dipping Method for Efficient and Stable Pure-Blue Electroluminescence. Adv. Mater. 2007, $19,2574-2579$.
(24) Grell, M.; Bradley, D. D. C.; Ungar, G.; Hill, J.; Whitehead, K. S. Interplay of Physical Structure and Photophysics for a Liquid Crystalline Polyfluorene. Macromolecules 1999, 32, 5810-5817.

(25) Ariu, M.; Sims, M.; Rahn, M. D.; Hill, J.; Fox, A. M.; Lidzey, D. G.; Oda, M.; Cabanillas-Gonzalez, J.; Bradley, D. D. C. Exciton Migration in $\beta$-Phase Poly(9,9-dioctylfluorene). Phys. Rev. B 2003, 67, 195333.

(26) Cheetham, N. J.; Ortiz, M.; Perevedentsev, A.; Dion-Bertrand, L.-I.; Greetham, G. M.; Sazanovich, I. V.; Towrie, M.; Parker, A. W.; Nelson, J.; Silva, C.; Bradley, D. D. C.; Hayes, S. C.; Stavrinou, P. N. The Importance of Microstructure in Determining Polaron Generation Yield in Poly(9,9-dioctylfluorene). Chem. Mater. 2019, 31, 6787-6797.

(27) Bright, D. W.; Dias, F. B.; Galbrecht, F.; Scherf, U.; Monkman, A. P. The Influence of Alkyl-Chain Length on Beta-Phase Formation in Polyfluorenes. Adv. Funct. Mater. 2009, 19, 67-73.

(28) Bright, D. W.; Moss, K. C.; Kamtekar, K. T.; Bryce, M. R.; Monkman, A. P. The $\beta$ Phase Formation Limit in Two Poly(9,9-di- $n-$ octylfluorene) based Copolymers. Macromol. Rapid Commun. 2011, 32, 983-987.

(29) Kuehne, A. J. C.; Mackintosh, A. R.; Pethrick, R. A. $\beta$-Phase Formation in a Crosslinkable Poly(9,9-dihexylfluorene). Polymer 2011, 52, 5538-5542.

(30) Hamilton, I.; Chander, N.; Cheetham, N. J.; Suh, M.; Dyson, M.; Wang, X.; Stavrinou, P. N.; Cass, M.; Bradley, D. D. C.; Kim, J.-S. Controlling Molecular Conformation for Highly Efficient and Stable Deep-Blue Copolymer Light-Emitting Diodes. ACS Appl. Mater. Interfaces 2018, 10, 11070-11082.

(31) Wilkinson, C. I.; Lidzey, D. G.; Palilis, L. C.; Fletcher, R. B.; Martin, S. J.; Wang, X. H.; Bradley, D. D. C. Enhanced Performance of Pulse Driven Small Area Polyfluorene Light Emitting Diodes. Appl. Phys. Lett. 2001, 79, 171-173.

(32) Belton, C. R.; Kanibolotsky, A. L.; Kirkpatrick, J.; Orofino, C.; Elmasly, S. E. T.; Stavrinou, P. N.; Skabara, P. J.; Bradley, D. D. C. Location, Location, Location - Strategic Positioning of 2,1,3Benzothiadiazole Units within Trigonal Quaterfluorene-Truxene Star-Shaped Structures. Adv. Funct. Mater. 2013, 23, 2792-2804.

(33) Karl, M.; Glackin, J. M. E.; Schubert, M.; Kronenberg, N. M.; Turnbull, G. A.; Samuel, I. D. W.; Gather, M. C. Flexible and UltraLightweight Polymer Membrane Lasers. Nat. Commun. 2018, 9, 1525. (34) Amarasinghe, D.; Ruseckas, A.; Vasdekis, A. E.; Turnbull, G. A.; Samuel, I. D. W. High-Gain Broadband Solid-State Optical Amplifier using a Semiconducting Copolymer. Adv. Mater. 2009, 21, 107-110.

(35) Demir, N.; Oner, I.; Varlikli, C.; Ozsoy, C.; Zafer, C. Efficiency Enhancement in a Single Emission Layer Yellow Organic Light Emitting Device: Contribution of CIS/ZnS Quantum Dot. Thin Solid Films 2015, 589, 153-160.

(36) Chappell, J.; Lidzey, D. G.; Jukes, P. C.; Higgins, A. M.; Thompson, R. L.; O’Connor, S.; Grizzi, I.; Fletcher, R.; O’Brien, J.; Geoghegan, M.; Jones, R. A. L. Correlating Structure with Fluorescence Emission in Phase-Separated Conjugated-Polymer Blends. Nat. Mater. 2003, 2, 616-621.

(37) Xia, R.; Stavrinou, P. N.; Bradley, D. D. C.; Kim, Y. Efficient Optical Gain Media Comprising Binary Blends of Poly(3-hexylthiophene) and Poly(9,9-dioctylfluorene-co-benzothiadiazole). J. Appl. Phys. 2012, 111, 123107.

(38) Winfield, J. M.; Van Vooren, A.; Park, M. J.; Hwang, D. H.; Cornil, J.; Kim, J.-S.; Friend, R. H. Charge-Transfer Character of Excitons in Poly[2,7-(9,9-di- $n$-octylfluorene) ${ }_{(1-x)^{-}}$-co-4,7-(2,1,3-benzothiadiazole $\left.)_{(x)}\right]$. J. Chem. Phys. 2009, 131, No. 035104.

(39) Xia, R.; Heliotis, G.; Hou, Y.; Bradley, D. D. C. Fluorene-Based Conjugated Polymer Optical Gain Media. Org. Electron. 2003, 4, 165-177.

(40) Köhler, A.; Bässler, H. Electronic Processes in Organic Semiconductors: An Introduction; Wiley-VCH: Weinheim, Germany, 2015.

(41) Kim, J.-S.; Friend, R. H.; Grizzi, I.; Burroughes, J. H. Spin-Cast Thin Semiconducting Polymer Interlayer for Improving Device 
Efficiency of Polymer Light-Emitting Diodes. Appl. Phys. Lett. 2005, 87, No. 023506.

(42) Shaheen, S. E.; Jabbour, G. E.; Morrell, M. M.; Kawabe, Y.; Kippelen, B.; Peyghambarian, N.; Nabor, M. F.; Schlaf, R.; Mash, E. A.; Armstrong, N. R. Bright Blue Organic Light-Emitting Diode with Improved Color Purity Using a LiF/Al Cathode. J. Appl. Phys. 1998, 84, 2324-2327.

(43) Forrest, S. R.; Bradley, D. D. C.; Thompson, M. E. Measuring the Efficiency of Organic Light-Emitting Devices. Adv. Mater. 2003, $15,1043-1048$.

(44) Arredondo, B.; Romero, B.; Gutiérrez-Llorente, A.; Martínez, A. I.; Álvarez, A. L.; Quintana, X.; Otón, J. M. On the Electrical Degradation and Green Band Formation in $\alpha$ - and $\beta$-Phase Poly(9,9dioctyfluorene) Polymer Light-Emitting Diodes. Solid-State Electron. 2011, 61, 46-52.

(45) Juška, G.; Nekrašas, N.; Genevičius, K. Investigation of Charge Carriers Transport from Extraction Current Transients of Injected Charge Carriers. J. Non-Cryst. Solids 2012, 358, 748-750.

(46) Armin, A.; Juska, G.; Ullah, M.; Velusamy, M.; Burn, P. L.; Meredith, P.; Pivrikas, A. Balanced Carrier Mobilities: Not a Necessary Condition for High-Efficiency Thin Organic Solar Cells as Determined by MIS-CELIV. Adv. Energy Mater. 2014, 4, 1300954.

(47) Bai, Z.; Liu, Y.; Li, T.; Li, X.; Liu, B.; Liu, B.; Lu, D. Quantitative Study on $\beta$-Phase Heredity Based on Poly(9,9dioctylfluorene) from Solutions to Films and the Effect on Hole Mobility. J. Phys. Chem. C 2016, 120, 27820-27828.

(48) Campbell, A. J.; Bradley, D. D. C.; Antoniadis, H. Dispersive Electron Transport in an Electroluminescent Polyfluorene Copolymer Measured by the Current Integration Time-of-Flight Method. Appl. Phys. Lett. 2001, 79, 2133-2135.

(49) Poplavskyy, D.; Nelson, J.; Bradley, D. D. C. Ohmic Hole Injection in Poly(9,9-dioctylfluorene) Polymer Light-Emitting Diodes. Appl. Phys. Lett. 2003, 83, 707-709.

(50) Tanase, C.; Meijer, E. J.; Blom, P. W. M.; De Leeuw, D. M. Unification of the Hole Transport in Polymeric Field-Effect Transistors and Light-Emitting Diodes. Phys. Rev. Lett. 2003, 91, 216601.

(51) Campbell, A. J.; Rawcliffe, R.; Guite, A.; Faria, J. C. D.; Mukherjee, A.; McLachlan, M. A.; Shkunov, M.; Bradley, D. D. C. Charge-Carrier Density Independent Mobility in Amorphous Fluorene-Triarylamine Copolymers. Adv. Funct. Mater. 2016, 26, $3720-3729$.

(52) Martens, H. C. F.; Brom, H. B.; Blom, P. W. M. FrequencyDependent Electrical Response of Holes in Poly ( $p$-phenylene vinylene). Phys. Rev. B 1999, 60, R8489-R8492.

(53) Martens, H. C. F.; Huiberts, J. N.; Blom, P. W. M. Simultaneous Measurement of Electron and Hole Mobilities in Polymer Light-Emitting Diodes. Appl. Phys. Lett. 2000, 77, 1852.

(54) Budzisz, P.; Signerski, R.; Jarosz, G. Effect of Shallow Traps on Admittance Spectra of the System Carrying SCLC and on Values of Charge Carrier Mobility Extracted from Susceptance. Chem. Phys. 2015, 456, 61-64.

(55) Sandberg, O. J.; Nyman, M.; Dahlström, S.; Sandén, S.; Törngren, B.; Smått, J.-H.; Österbacka, R. On the Validity of MISCELIV for Mobility Determination in Organic Thin-Film Devices. Appl. Phys. Lett. 2017, 110, 153504.

(56) Tsang, S. W.; So, S. K.; Xu, J. B. Application of Admittance Spectroscopy to Evaluate Carrier Mobility in Organic Charge Transport Materials. J. Appl. Phys. 2006, 99, No. 013706.

(57) Weiß, O. J.; Krause, R. K.; Hunze, A. Hole Mobility of 1NaphDATA. J. Appl. Phys. 2008, 103, No. 043709.

(58) Campbell, A. J.; Bradley, D. D. C.; Lidzey, D. G. Space-Charge Limited Conduction with Traps in Poly(phenylene vinylene) Light Emitting Diodes. J. Appl. Phys. 1997, 82, 6326-6342.

(59) Cornil, J.; Gueli, I.; Dkhissi, A.; Sancho-Garcia, J. C.; Hennebicq, E.; Calbert, J. P.; Lemaur, V.; Beljonne, D.; Brédas, J. L. Electronic and Optical Properties of Polyfluorene and FluoreneBased Copolymers: A Quantum-Chemical Characterization. J. Chem. Phys. 2003, 118, 6615-6623.
(60) Foster, S. On the Influence of Physical and Chemical Structure on Charge Transport in Disordered Semiconducting Materials and Devices; PhD thesis, Imperial College London, UK, January 2013.

(61) O'Carroll, D.; Iacopino, D.; O’Riordan, A.; Lovera, P.; O'Connor, E.; O’Brien, G. A.; Redmond, G. Poly(9,9-dioctylfluorene) Nanowires with Pronounced $\beta$-Phase Morphology: Synthesis, Characterization, and Optical Properties. Adv. Mater. 2008, 20, 4248.

(62) van Mensfoort, S. L. M.; Coehoorn, R. Determination of Injection Barriers in Organic Semiconductor Devices from Capacitance Measurements. Phys. Rev. Lett. 2008, 100, No. 086802.

(63) Yi, G. R.; Kim, H. S.; Jeong, K. W.; Kim, C. K. The Effect of Bilayer Hole Transporting Layers Using Thermal Crosslinking Technology on the Characteristics of Organic Light-Emitting Diodes. Mol. Cryst. Liq. Cryst. 2017, 651, 99-107.

(64) Amruth, C.; Szymański, M. Z.; Kuszczyńska, B.; Ulański, J. Inkjet Printing of Super Yellow: Ink Formulation, Film Optimization, OLEDs Fabrication, and Transient Electroluminescence. Sci. Rep. 2019, 9, 8493.

(65) Knapp, E.; Ruhstaller, B. Numerical Impedance Analysis for Organic Semiconductors with Exponential Distribution of Localized States. Appl. Phys. Lett. 2011, 99, No. 093304.

(66) Giebeler, C.; Whitelegg, S. A.; Lidzey, D. G.; Lane, P. A.; Bradley, D. D. C. Device Degradation of Polymer Light Emitting Diodes Studied by Electroabsorption Measurements. Appl. Phys. Lett. 1999, 75, 2144-2146.

(67) Kawano, K.; Pacios, R.; Poplavskyy, D.; Nelson, J.; Bradley, D. D. C.; Durrant, J. R. Degradation of Organic Solar Cells due to Air Exposure. Sol. Energy Mater. Sol. Cells 2006, 90, 3520-3530.

(68) Donley, C. L.; Zaumseil, J.; Andreasen, J. W.; Nielsen, M. M.; Sirringhaus, H.; Friend, R. H.; Kim, J.-S. Effects of Packing Structure on the Optoelectronic and Charge Transport Properties in Poly(9,9di-n-octylfluorene-alt-benzothiadiazole). J. Am. Chem. Soc. 2005, 127, 12890-12899.

(69) Sims, M.; Bradley, D. D. C.; Ariu, M.; Koeberg, M.; Asimakis, A.; Grell, M.; Lidzey, D. G. Understanding the Origin of the $535 \mathrm{~nm}$ Emission Band in Oxidized Poly(9,9-dioctylfluorene): The Essential Role of Inter-Chain/Inter-Segment Interactions. Adv. Funct. Mater. 2004, 14, 765-781. 